\title{
Logarithm Laws for Equilibrium States in Negative Curvature
}

\author{
Frédéric Paulin ${ }^{1}$, Mark Pollicott ${ }^{2}$ \\ 1 Département de Mathématique, Bât. 425, UMR 8628 CNRS Université Paris-Sud, 91505 ORSAY Cedex, \\ France E-mail: frederic.paulin@math.u-psud.fr \\ 2 Mathematics Institute, University of Warwick, Coventry CV4 7AL, UK \\ E-mail: masdbl@warwick.ac.uk
}

Received: 9 May 2014 / Accepted: 29 January 2015

Published online: 5 July 2016 - ( ) The Author(s) 2016. This article is published with open access

at Springerlink.com

\begin{abstract}
Let $M$ be a pinched negatively curved Riemannian manifold, whose unit tangent bundle is endowed with a Gibbs measure $m_{F}$ associated with a potential $F$. We compute the Hausdorff dimension of the conditional measures of $m_{F}$. We study the $m_{F}$-almost sure asymptotic penetration behaviour of locally geodesic lines of $M$ into small neighbourhoods of closed geodesics, and of other compact (locally) convex subsets of $M$. We prove Khintchine-type and logarithm law-type results for the spiraling of geodesic lines around these objects. As an arithmetic consequence, we give almost sure Diophantine approximation results of real numbers by quadratic irrationals with respect to general Hölder quasi-invariant measures.
\end{abstract}

\section{Introduction}

Let $M$ be a complete connected Riemannian manifold with pinched sectional curvature at most -1 , and let $\left(g^{t}\right)_{t \in \mathbb{R}}$ be its geodesic flow. In this paper, we consider for instance a closed geodesic $D_{0}$ in $M$, and we want to study the spiraling of geodesics lines around $D_{0}$. Given an ergodic probability measure $m$ invariant under $\left(g^{t}\right)_{t \in \mathbb{R}}$, whose support is the nonwandering set $\Omega$ of the geodesic flow, $m$-almost every orbit is dense in $\Omega$. Two geodesic lines, having at some time their unit tangent vectors very close, remain close for a long time. Hence $m$-almost every geodesic line will stay for arbitrarily long periods of time in a given small neighbourhood of $D_{0}$. In what follows, we make this behaviour quantitative when $m$ is any equilibrium state.

Let $F: T^{1} M \rightarrow \mathbb{R}$ be a potential, that is, a Hölder-continuous function. Let $\mathscr{M}$ be the set of probability measures $m$ on $T^{1} M$ invariant under the geodesic flow, for which the negative part of $F$ is $m$-integrable, and let $h_{m}\left(g^{1}\right)$ be the (metric) entropy of the geodesic flow with respect to $m$. The pressure of the potential $F$ is

$$
P=P(F)=\sup _{m \in \mathscr{M}}\left(h_{m}\left(g^{1}\right)+\int_{T^{1} M} F d m\right) .
$$


Let $m_{F}$ be a Gibbs measure on $T^{1} M$ associated with the potential $F$ (see [PPS] and Sect. 2 ). When finite and normalised to be a probability measure (and if the negative part of $F$ is $m_{F}$-integrable and the sectional curvature of $M$ has bounded first order derivatives), it is the unique equilibrium state, that is, it attains the upper bound defining the pressure $P(F)$ (see [PPS, Theo. 6.1], improving [OP] when $F=0$ ). For instance, $m_{F}$ is (up to a constant multiple) the Bowen-Margulis measure $m_{\mathrm{BM}}$ if $F=0$, and is the Liouville measure if $F$ is the strong unstable Jacobian $v \mapsto-\left.\frac{d}{d t}\right|_{t=0} \log \mathrm{Jac}\left(g^{t}{ }_{\mid W^{\mathrm{su}}(v)}\right)(v)$ and $M$ is compact (see [PPS, Theo. 7.2] for a more general situation). We will use the construction of $m_{F}$ by Paulin et al. [PPS] (building on work of Ledrappier [Led1,Led2], Hamenstädt [Ham1,Ham2] especially for the harmonic measures, Coudène [Cou], Mohsen [Moh]) via Patterson densities $\left(\mu_{x}^{F}\right)_{x \in \widetilde{M}}$ on the boundary at infinity $\partial_{\infty} \widetilde{M}$ of a universal cover $\widetilde{M}$ of $M$ associated with the potential $F$.

We first prove (see Sect. 3) the following result relating measure theoretic invariants of $m_{F}$ and $\mu_{x}^{F}$, which extends Ledrappier's result [Led4, $\left.\S 4\right]$ when $F=0$.

Theorem 1.1. If $m_{F}$ is finite and $F$ is $m_{F}$-integrable, if the sectional curvature of $M$ has bounded first order derivatives, then the Hausdorf dimension of the Patterson measure $\mu_{x}^{F}$ (with respect to the Gromov-Bourdon visual distance on $\partial_{\infty} \widetilde{M}$ ) is equal to the metric entropy of the Gibbs measure $m_{F}$ (for the geodesic flow).

Let $D_{0}$ be a closed geodesic in $M$ of length $\ell_{0}$. If $v_{0} \in T^{1} M$ is tangent to $D_{0}$, let

$$
P_{0}=P\left(F_{\mid T^{1} D_{0}}\right)=\frac{\max \left\{\int_{0}^{\ell_{0}} F\left(g^{t} v_{0}\right) d t, \int_{0}^{\ell_{0}} F\left(g^{t}\left(-v_{0}\right)\right) d t\right\}}{\ell_{0}} .
$$

We will prove that $P_{0}<P$ if $m_{F}$ is finite. Let $\epsilon_{0}>0$ and let $\psi:[0,+\infty[\rightarrow[0,+\infty[$ be a Lipschitz map. As introduced in [HP2], let $E(\psi)$ be the set of $\left(\epsilon_{0}, \psi\right)$-Liouville vectors around $D_{0}$, that is, the set of $v \in T^{1} M$ such that there exists a sequence $\left(t_{n}\right)_{n \in \mathbb{N}}$ in $\left[0,+\infty\right.$ [ converging to $+\infty$ such that for every $t \in\left[t_{n}, t_{n}+\psi\left(t_{n}\right)\right]$, the footpoint of $g^{t} v$ belongs to the $\epsilon_{0}$-neighbourhood $\mathscr{N}_{\epsilon_{0}} D_{0}$ of $D_{0}$.

The Khintchine-type result describing the spiraling around the closed geodesic $D_{0}$ is the following (simplified version of the) main result of this paper (see Sect. 4).

Theorem 1.2. Assume that $M$ is compact. If the integral $\int_{0}^{+\infty} e^{\psi(t)\left(P_{0}-P\right)} d t$ diverges (resp. converges) then $m_{F}$-almost every (resp. no) point of $T^{1} M$ belongs to $E(\psi)$.

When $F=0$ (that is, when $m_{F}$ is the Bowen-Margulis measure), this result is due to Hersonsky and Paulin [HP2]. As $m_{F}$ can be taken to be the Liouville measure, this theorem answers a question raised in loc. cit. This result, in this particular case when $D_{0}$ is a closed geodesic, can be restated as a well-approximation type of result of points in the limit set of the fundamental group of $\Gamma$ by an orbit of a loxodromic fixed point, see for instance [FSU] for very general results (their measure on the limit set corresponds to $F=0$, though an extension might be possible), and the references of [FSU] for historical motivation and partial results. This result is a shrinking target problem type, and our main tool is the mixing property of the geodesic flow of $M$ for Gibbs measure (see [PPS]).

We stated this result as such to emphasize its novelty even in the compact case, but it is true in a much more general setting, both from $M$ and $D_{0}$ (see Theorem 4.1). For instance, when $M$ is a geometrically finite locally symmetric orbifold, when $F$ has finite pressure $P(F)$ and finite Gibbs measure $m_{F}$, when $D_{0}$ is a compact totally geodesic 
suborbifold (of positive dimension and codimension), the result still holds. When $M$ is the quotient of the real hyperbolic 3-space by a geometrically finite Kleinian group $\Gamma$, when $F$ has finite pressure $P(F)$ and finite Gibbs measure $m_{F}$, and when $D_{0}$ is the convex hull of the limit set of a precisely invariant quasi-fuschian closed surface subgroup $\Gamma_{0}$ of $\Gamma$, the result still holds. See Sect. 4 for more examples.

When $F=0$, the following logarithm law for the almost sure spiraling of geodesic lines around $D_{0}$ is due to Hersonsky and Paulin [HP2]. Let $\pi: T^{1} M \rightarrow M$ be the unit tangent bundle. Define the penetration map $\mathfrak{p}: T^{1} M \times \mathbb{R} \rightarrow[0,+\infty]$ of the geodesic lines inside $\mathscr{N}_{\epsilon_{0}} D_{0}$ by $\mathfrak{p}(v, t)=0$ if $\pi\left(\phi_{t} v\right) \notin \mathscr{N}_{\epsilon_{0}} D_{0}$, and otherwise $\mathfrak{p}(v, t)$ is the maximal length of an interval $I$ in $\mathbb{R}$ containing $t$ such that $\pi\left(\phi_{s} v\right) \in \mathscr{N}_{\epsilon_{0}} D_{0}$ for every $s \in I$.

Corollary 1.3. Under the assumptions of Theorem 1.2, for $m_{F}$-almost every $v \in T^{1} M$, we have

$$
\limsup _{t \rightarrow+\infty} \frac{\mathfrak{p}(v, t)}{\log t}=\frac{1}{P-P_{0}} .
$$

In Sect. 5, we will give arithmetic applications of Theorem 1.2. We will in particular generalise to a huge class of measures on $\mathbb{R}$ the Khintchine-type result of approximation of real numbers by quadratic irrationals over $\mathbb{Q}$, which was proved in [PaP2] for the Lebesgue measure, and prove other 0-1-laws of approximations of real numbers by arithmetically defined points. To conclude this introduction, we give one example of such a result.

Let $a, b \in \mathbb{N}-\{0\}$ be positive integers such that the equation $x^{2}-a y^{2}-b z^{2}=0$ has no nonzero integer solution (for instance $a=2$ and $b=3$ ). Let $\Gamma_{a, b}$ be

$$
\left\{\left(\begin{array}{cc}
x+y \sqrt{a} & z-t \sqrt{a} \\
b(z+t \sqrt{a}) & x-y \sqrt{a}
\end{array}\right):(x, y, z, t) \in \mathbb{Z}^{4} \text { and } x^{2}-a y^{2}-b z^{2}+a b t^{2}=1\right\},
$$

which is a discrete subgroup of $\mathrm{SL}_{2}(\mathbb{R})$, whose action by homographies on $\mathbb{P}_{1}(\mathbb{R})=$ $\mathbb{R} \cup\{\infty\}$ is denoted by $\cdot$. If $\alpha \in \mathbb{R}$ is a solution of the equation $\gamma \cdot X=X$ for some $\gamma \in \Gamma_{a}, b$, then $\alpha$ is quadratic over $\mathbb{Q}(\sqrt{a})$, and if furthermore $\alpha \notin \mathbb{Q}(\sqrt{a})$, we denote by $\alpha^{\sigma}$ its Galois conjugate over $\mathbb{Q}(\sqrt{a})$. Given $\gamma \in \Gamma_{a}, b$ with $\operatorname{trace} \operatorname{tr} \gamma \neq 0, \pm 2$, we denote by $\gamma^{+}$and $\gamma^{-}$the attractive and repulsive fixed points of $\gamma$ in $\mathbb{R} \cup\{\infty\}$.

Given a continuous action of a discrete group $G$ on a compact metric space $(X, d)$, recall that a Hölder quasi-invariant measure (see for instance [Led3,Ham2]) on $X$ (for the action of $G$ ) is a probability measure $\mu$ such that for every $g \in G$, the measure $g_{*} \mu$ is absolutely continuous with respect to $\mu$, and the Radon-Nykodim derivative $\frac{d g_{*} \mu}{d \mu}$ coincides $\mu$-almost everywhere with a Hölder-continuous map on $X$, which we will still denote by $\frac{d g_{*} \mu}{d \mu}$.

The next result is a Khintchine-type of result, under a huge class of measures, for the Diophantine approximation of real numbers by quadratic irrationals over $\mathbb{Q}(\sqrt{a})$ in a (dense) orbit under the arithmetic group $\Gamma_{a, b}$ (extended by the Galois conjugation).

Corollary 1.4. Let $\mu$ be a Hölder quasi-invariant measure on $\mathbb{R} \cup\{\infty\}$ for the action by homographies of $\Gamma_{a, b}$. Let $\gamma_{0}$ be a primitive element in $\Gamma_{a, b}$ with $\operatorname{tr}\left(\gamma_{0}\right) \neq 0, \pm 2$. For $\mu$-almost every $x \in \mathbb{R}$, we have

$$
\liminf _{\alpha \in \Gamma_{a, b} \cdot\left\{\gamma_{0}^{-}, \gamma_{0}^{+}\right\},\left|\alpha-\alpha^{\sigma}\right| \rightarrow 0} \frac{|x-\alpha|}{\left|\alpha-\alpha^{\sigma}\right|\left(-\log \left|\alpha-\alpha^{\sigma}\right|\right)^{-s}}=0 \quad(\text { resp. }=+\infty)
$$


if $s \leq \frac{1}{\delta-\delta_{0}}\left(\right.$ resp. $\left.s>\frac{1}{\delta-\delta_{0}}\right)$, where

$$
\delta=\limsup _{s \rightarrow+\infty} \frac{1}{2 \log s} \log \sum_{\gamma \in \Gamma_{a, b}, 2<|\operatorname{tr}(\gamma)| \leq s} \frac{d\left(\gamma^{-1}\right)_{*} \mu}{d \mu}\left(\gamma^{+}\right)
$$

and $\delta_{0}=\frac{1}{2 \operatorname{arcosh}\left(\frac{\left|\operatorname{tr} \gamma_{0}\right|}{2}\right)} \max \left\{\frac{d\left(\gamma_{0}^{-1}\right)_{*} \mu}{d \mu}\left(\gamma_{0}^{+}\right), \frac{d\left(\gamma_{0}\right)_{*} \mu}{d \mu}\left(\gamma_{0}^{-}\right)\right\}$.

We refer to Sect. 5 for more general results, in particular for approximations with congruence properties and for the approximation of complex numbers by quadratic irrationals over an imaginary quadratic extension of $\mathbb{Q}$.

\section{A Summary of the Patterson-Sullivan Theory for Gibbs States}

Most of the content of this section is extracted from [PPS], to which we refer for the proofs of the claims and for more details.

Let $\widetilde{M}$ be a complete simply connected Riemannian manifold with (dimension at least 2 and) pinched negative sectional curvature $-b^{2} \leq K \leq-1$, and let $x_{0} \in \widetilde{M}$ be a fixed basepoint. For every $\epsilon>0$ and every subset $A$ of $\widetilde{M}$, we denote by $\mathscr{N}_{\epsilon} A$ the closed $\epsilon$-neighbourhood of $A$ in $\widetilde{M}$.

We denote by $\pi: T^{1} \widetilde{M} \rightarrow \widetilde{M}$ the unit tangent bundle of $\widetilde{M}$, where $T^{1} \widetilde{M}$ is endowed with Sasaki's Riemannian metric. Let $\partial_{\infty} \widetilde{M}$ be the boundary at infinity of $\widetilde{M}$. We denote by $\Lambda G$ the limit set of any discrete group of isometries $G$ of $\widetilde{M}$, and by $\mathscr{C} \Lambda G$ the convex hull in $\widetilde{M}$ of $\Lambda G$, if $\Lambda G$ has at least two elements.

Let $\Gamma$ be a nonelementary (not virtually nilpotent) discrete group of isometries of $\widetilde{M}$. Let $M$ and $T^{1} M$ be the quotient Riemannian orbifolds $\Gamma \backslash \widetilde{M}$ and $\Gamma \backslash T^{1} \widetilde{M}$, and let again $\pi: T^{1} M \rightarrow M$ be the map induced by $\pi: T^{1} \widetilde{M} \rightarrow \widetilde{M}$. We denote by $\left(g^{t}\right)_{t \in \mathbb{R}}$ the geodesic flow on $T^{1} \widetilde{M}$, as well as its quotient flow on $T^{1} M$.

For every $v \in T^{1} \widetilde{M}$, let $v_{-} \in \partial_{\infty} \widetilde{M}$ and $v_{+} \in \partial_{\infty} \widetilde{M}$, respectively, be the endpoints at $-\infty$ and $+\infty$ of the geodesic line $g_{v}: \mathbb{R} \rightarrow \widetilde{M}$ defined by $v$ (that is, such that $\dot{g_{v}}(0)=v$ ). Let $\partial_{\infty}^{2} \widetilde{M}$ be the subset of $\partial_{\infty} \dot{\widetilde{M}} \times \partial_{\infty} \widetilde{M}$ which consists of pairs of distinct points at infinity of $\widetilde{M}$. Hopf's parametrisation of $T^{1} \widetilde{M}$ is the homeomorphism which identifies $T^{1} \widetilde{M}$ with $\partial_{\infty}^{2} \widetilde{M} \times \mathbb{R}$, by the map $v \mapsto\left(v_{-}, v_{+}, t\right)$, where $t$ is the signed distance of the closest point to $x_{0}$ on $g_{v}(\mathbb{R})$ to $\pi(v)$. Let $\widetilde{\Omega} \Gamma$ be the $\Gamma$-invariant set of $v \in T^{1} \widetilde{M}$ such that $v_{-}, v_{+} \in \Lambda \Gamma$, whose image in $T^{1} M$ is the nonwandering set of the geodesic flow of $T^{1} M$.

Let $\iota: T^{1} \widetilde{M} \rightarrow T^{1} \widetilde{M}$ be the (Hölder-continuous) antipodal (flip) map of $T^{1} \tilde{M}$ defined by $\iota v=-v$. We denote the quotient map of $\iota$ again by $\iota: T^{1} M \rightarrow T^{1} M$.

Let $\widetilde{F}: T^{1} \widetilde{M} \rightarrow \mathbb{R}$ be a fixed Hölder-continuous $\Gamma$-invariant function, called a potential on $T^{1} \widetilde{M}$. It induces a Hölder-continuous function $F: T^{1} M \rightarrow \mathbb{R}$, called a potential on $T^{1} M$. Two potentials $\widetilde{F}$ and $\widetilde{F}^{*}$ on $T^{1} \widetilde{M}$ (or their induced maps on $T^{1} M$ ) are cohomologous if there exists a Hölder-continuous $\Gamma$-invariant map $\widetilde{G}: T^{1} \widetilde{M} \rightarrow \mathbb{R}$, differentiable along every flow line, such that

$$
\widetilde{F}^{*}(v)-\widetilde{F}(v)=\frac{d}{d t}{ }_{\mid t=0} \widetilde{G}\left(\phi_{t} v\right) .
$$

For any two distinct points $x, y \in \widetilde{M}$, let $v_{x y} \in T_{x}^{1} \tilde{M}$ be the initial tangent vector of the oriented geodesic segment $[x, y]$ in $\widetilde{M}$ that connects $x$ to $y$; define 


$$
\int_{x}^{y} \widetilde{F}=\int_{0}^{d(x, y)} \widetilde{F}\left(g^{t} v_{x y}\right) d t
$$

and $\int_{x}^{x} \widetilde{F}=0$ for all $x \in \widetilde{M}$. Given a hyperbolic element $\gamma \in \Gamma$ with translation axis $A_{\gamma}$, the period of $\gamma$ for $F$ is, for any $x \in A_{\gamma}$,

$$
\operatorname{Per}_{F}(\gamma)=\int_{x}^{\gamma x} \widetilde{F}
$$

The critical exponent of $(\Gamma, F)$ is

$$
\delta_{\Gamma, F}=\limsup _{n \rightarrow+\infty} \frac{1}{n} \log \sum_{\gamma \in \Gamma, n-1<d(x, \gamma y) \leq n} e^{\int_{x}^{\gamma y} \widetilde{F}} .
$$

When $F=0$, then $\delta_{\Gamma, F}$ is the standard critical exponent $\delta_{\Gamma}$ of $\Gamma$. Note that

$$
\int_{x}^{y} \widetilde{F}=\int_{y}^{x} \widetilde{F} \circ \iota
$$

that $\delta_{\Gamma, F}=\delta_{\Gamma, F \circ \iota}>-\infty$ and that $\delta_{\Gamma, F+c}=\delta_{\Gamma, F}+c$ for every constant $c>0$ (see [PPS, Lem. 3.3]). We assume that $\delta_{\Gamma, F}<+\infty$ (this is for instance satisfied if $\widetilde{F}$ is bounded, see [PPS, Lem. 3.3]). By [PPS, Theo. 6.1], if the sectional curvature of $\widetilde{M}$ has bounded first order derivatives, then the critical exponent $\delta_{\Gamma, F}$ is equal to the pressure $P(F)$ of $F$ on $T^{1} M$, defined in Eq. (1). The Poincaré series

$$
Q_{\Gamma, F, x_{0}}(s)=\sum_{\gamma \in \Gamma} e^{\int_{x_{0}}^{\gamma x_{0}}(\widetilde{F}-s)}
$$

of $(\Gamma, F)$ converges if $s>\delta_{\Gamma, F}$ and diverges if $s<\delta_{\Gamma, F}$. We say that $(\Gamma, F)$ is of divergence type if $Q_{\Gamma, F, x_{0}}(s)$ diverges at $s=\delta_{\Gamma, F}$.

The (normalised) Gibbs cocycle of $\widetilde{F}$ is the function $C^{F}: \partial_{\infty} \widetilde{M} \times \widetilde{M} \times \widetilde{M} \rightarrow \mathbb{R}$ defined by

$$
(\xi, x, y) \mapsto C_{\xi}^{F}(x, y)=\lim _{t \rightarrow+\infty} \int_{y}^{\xi_{t}}\left(\widetilde{F}-\delta_{\Gamma, F}\right)-\int_{x}^{\xi_{t}}\left(\widetilde{F}-\delta_{\Gamma, F}\right),
$$

where $t \mapsto \xi_{t}$ is any geodesic ray with endpoint $\xi \in \partial_{\infty} \widetilde{M}$. We have $C^{F+c}=C^{F}$ for every constant $c \in \mathbb{R}$. If $\widetilde{F}=0$, then $C^{F}=\delta_{\Gamma} \beta$, where $\beta$ is the Busemann cocycle.

By [PPS, Lem. 3.2, 3.4], there exists a constant $c_{1}>0$ (depending only on the Hölder constants of $\widetilde{\widetilde{F}}$ and on the bounds of the sectional curvature of $\widetilde{M}$ ) such that for all $x, y, z \in \widetilde{M}$, we have

$$
\left|\int_{x}^{z} \widetilde{F}-\int_{y}^{z} \widetilde{F}\right| \leq c_{1} e^{d(x, y)}+d(x, y) \max _{\pi^{-1}(B(x, d(x, y)))}|\widetilde{F}|,
$$

and, for every $\xi \in \partial_{\infty} \widetilde{M}$,

$$
\left|C_{\xi}^{F}(x, y)\right| \leq c_{1} e^{d(x, y)}+d(x, y) \max _{\pi^{-1}(B(x, d(x, y)))}|\widetilde{F}| .
$$


A family $\left(\mu_{x}^{F}\right)_{x \in \widetilde{M}}$ of finite measures on $\partial_{\infty} \widetilde{M}$, whose support is the limit set $\Lambda \Gamma$ of $\Gamma$, is a Patterson density for the pair $(\Gamma, \widetilde{F})$ if

$$
\gamma_{*} \mu_{x}^{F}=\mu_{\gamma x}^{F}
$$

for all $\gamma \in \Gamma$ and $x \in \widetilde{M}$, and if the following Radon-Nikodym derivatives exist for all $x, y \in \widetilde{M}$ and satisfy for almost all $\xi \in \partial_{\infty} \widetilde{M}$

$$
\frac{d \mu_{x}^{F}}{d \mu_{y}^{F}}(\xi)=e^{-C_{\xi}^{F}(x, y)} .
$$

A Gibbs measure on $T^{1} \widetilde{M}$ for $(\Gamma, \widetilde{F})$ is the measure $\widetilde{m}_{F}$ on $T^{1} \widetilde{M}$ given by the density

$$
d \widetilde{m}_{F}(v)=e^{C_{v_{-}}^{F \circ l}\left(x_{0}, \pi(v)\right)+C_{v_{+}}^{F}\left(x_{0}, \pi(v)\right)} d \mu_{x_{0}}^{F \circ \iota}\left(v_{-}\right) d \mu_{x_{0}}^{F}\left(v_{+}\right) d t
$$

in Hopf's parametrisation. Patterson densities $\left(\mu_{x}^{F}\right)_{x \in \widetilde{M}}$ and $\left(\mu_{x}^{F \circ \iota}\right)_{x \in \widetilde{M}}$ exist (see [PPS, $\S 3.6]$, their construction, whence the existence of $\widetilde{m}_{F}$, requires only $\Gamma$ to be nonelementary and $\left.\delta_{\Gamma, F}<+\infty\right)$. The Gibbs measure $\tilde{m}_{F}$ is independent of $x_{0}$, its support is $\widetilde{\Omega} \Gamma$, and it is invariant under the actions of the group $\Gamma$ and of the geodesic flow. Thus (see [PPS, §2.6]), it defines a measure $m_{F}$ on $T^{1} M$ which is invariant under the quotient geodesic flow, called a Gibbs measure on $T^{1} M$. For every constant $c>0$, note that $\left(\mu_{x}^{F}\right)_{x \in \widetilde{M}}$ is also a Patterson density for the pair $(\Gamma, \widetilde{F}+c)$, thus $\widetilde{m}_{F}$ is also a Gibbs measure for $(\Gamma, \widetilde{F}+c)$. If $m_{F}$ is finite, then the Patterson densities are unique up to a common multiplicative constant (see [PPS, §5.3]); hence the Gibbs measure $m_{F}$ is uniquely defined, up to a multiplicative constant, and, when normalised to be a probability measure, it is the unique equilibrium state for the potential $F$, if the negative part of $F$ is $m_{F}$-integrable and if the sectional curvature of $\widetilde{M}$ has bounded first order derivatives, see [PPS, Theo. 6.1].

By its definition as a quasi-product, the Gibbs measure $\widetilde{m}_{F}$ satisfies the following property, used without mention in what follows: for every $x \in \widetilde{M}$, the preimage by $v \mapsto v_{+}$of a set of measure 0 (respectively $>0$ ) for $\mu_{x}^{F}$ has measure 0 (respectively $>0$ ) for $\tilde{m}_{F}$.

We refer to [PPS, §8] for finiteness criteria of $m_{F}$, in particular satisfied if $M$ is compact. Babillot [Bab, Thm. 1] showed that if $m_{F}$ is finite, then it is mixing for the geodesic flow on $T^{1} M$ if the length spectrum of $\Gamma$ is nonarithmetic (that is, if the set of translation lengths of the elements of $\Gamma$ is not contained in a discrete subgroup of $\mathbb{R}$ ). This condition, conjecturally always true, is known, for example, if $\Gamma$ has a parabolic element, if $\Lambda \Gamma$ is not totally disconnected (hence if $M$ is compact), or if $\widetilde{M}$ is a surface or a (rank-one) symmetric space, see for instance [Dal1, Dal2].

For every subset $A$ of $\widetilde{M}$ and every point $x$ in $\widetilde{M} \cup \partial_{\infty} \widetilde{M}$, the shadow of $A$ seen from $x$ is the set $\mathscr{O}_{x} A$ of points at infinity of the geodesic rays or lines starting from $x$ and meeting A. By Mohsen's shadow lemma (see [PPS, Lem. 3.10]), for every $x, y \in \widetilde{M}$, if $R>0$ is large enough, there exists $c=c(R)>0$ such that for every $\gamma \in \Gamma$, we have

$$
\frac{1}{c} e^{\int_{x}^{\gamma y}\left(\widetilde{F}-\delta_{\Gamma, F}\right)} \leq \mu_{x}^{F}\left(\mathscr{O}_{x}(B(\gamma y, R))\right) \leq c e^{\int_{x}^{\gamma y}\left(\widetilde{F}-\delta_{\Gamma, F}\right)} .
$$

Here is a new consequence of Mohsen's shadow lemma which will be useful in this paper. Recall that a discrete group $G$ of isometries of $\widetilde{M}$ is convex-cocompact if its limit set $\Lambda G$ contains at least two points, and if the action of $G$ on the convex hull $\mathscr{C} \Lambda G$ in $\widetilde{M}$ of $\Lambda G$ has compact quotient. 
Lemma 2.1. Let $\Gamma_{0}$ be a convex-cocompact subgroup of $\Gamma$ such that $\delta_{\Gamma_{0}}, F_{0}<\delta_{\Gamma, F}$, where $F_{0}: \Gamma_{0} \backslash T^{1} \widetilde{M} \rightarrow \mathbb{R}$ is the map induced by $\widetilde{F}$. Then $\mu_{x_{0}}^{F}\left(\Lambda \Gamma_{0}\right)=0$.

Proof. Since $\Gamma_{0}$ is convex-cocompact, if $R$ is big enough, for every $n \in \mathbb{N}$, we have

$$
\Lambda \Gamma_{0} \subset \bigcup_{\gamma \in \Gamma_{0}, d\left(x_{0}, \gamma x_{0}\right) \geq n} \mathscr{O}_{x_{0}}\left(B\left(\gamma x_{0}, R\right)\right) .
$$

Hence, by Eq. (8), there exists $c>0$ such that for every $n \in \mathbb{N}$,

$$
\mu_{x_{0}}^{F}\left(\Lambda \Gamma_{0}\right) \leq \sum_{\gamma \in \Gamma_{0}, d\left(x_{0}, \gamma x_{0}\right) \geq n} \mu_{x_{0}}^{F}\left(\mathscr{O}_{x_{0}}\left(B\left(\gamma x_{0}, R\right)\right)\right) \leq c \sum_{\gamma \in \Gamma_{0}, d\left(x_{0}, \gamma x_{0}\right) \geq n} e^{\int_{x_{0}}^{\gamma x_{0}}\left(\widetilde{F}-\delta_{\Gamma, F}\right)} .
$$

The Poincaré series $Q_{\Gamma_{0}, F_{0}, x_{0}}\left(\delta_{\Gamma, F}\right)$ converges, as $\delta_{\Gamma_{0}, F_{0}}<\delta_{\Gamma, F}$. Since the remainder of a converging series tends to 0 , this proves the result.

A parabolic subgroup of $\Gamma$ is a maximal infinite subgroup $\Gamma_{0}$ of $\Gamma$ whose limit set $\Lambda \Gamma_{0}$ is a singleton. It is bounded if $\Gamma_{0} \backslash\left(\Lambda \Gamma-\Lambda \Gamma_{0}\right)$ is compact. For every bounded parabolic subgroup $\Gamma_{0}$, if $\Lambda \Gamma_{0}=\left\{\xi_{0}\right\}$, there exists (see for instance [Bow]) a unique $\Gamma$-equivariant family $\left(\mathscr{H}_{\alpha \xi_{0}}\right)_{\alpha \in \Gamma / \Gamma_{0}}$ of maximal closed horoballs in $\widetilde{M}$ with pairwise disjoint interiors and with $\mathscr{H}_{\xi_{0}}$ centred at $\xi_{0}$. The horoball $\mathscr{H}_{\xi_{0}}$ is precisely invariant under $\Gamma$, that is, its stabiliser in $\Gamma$ is $\Gamma_{0}$ and the inclusion $\stackrel{\circ}{\mathscr{H}_{\xi_{0}}} \subset \widetilde{M}$ induces an injection $\Gamma_{0} \backslash \stackrel{\circ}{\mathscr{H}}_{\xi_{0}} \rightarrow \Gamma \backslash \tilde{M}$. Note that if $\Gamma_{0}$ is a parabolic subgroup of $\Gamma$ and if $m_{F}$ is finite, then we also have $\mu_{x_{0}}^{F}\left(\Lambda \Gamma_{0}\right)=0$ (see [PPS, Prop. 5.13 (i)]).

\section{Hausdorff Dimension of Patterson Measures of Potentials}

Let $\widetilde{M}$ be a complete simply connected Riemannian manifold with pinched negative sectional curvature at most -1 having bounded first order derivatives. Let $\Gamma$ be a nonelementary discrete group of isometries of $\widetilde{M}$. Let $\widetilde{F}: T^{1} \widetilde{M} \rightarrow \mathbb{R}$ be a Hölder-continuous $\Gamma$-invariant function. Assume that $\delta=\delta_{\Gamma, F}$ is finite. Let $\widetilde{m}_{F}$ be the Gibbs measure on $T^{1} \widetilde{M}$ associated with a pair of Patterson densities $\left(\left(\mu_{x}^{F \circ \iota}\right)_{x \in \tilde{M}},\left(\mu_{x}^{F}\right)_{x \in \widetilde{M}}\right)$ for $(\Gamma, F \circ \iota)$ and $(\Gamma, F)$. We use the notation introduced in Sect. 2 .

We fix in this section a point $x$ in $\widetilde{M}$. We denote by $d_{x}$ the Gromov-Bourdon visual distance on $\partial_{\infty} \widetilde{M}$ seen from $x$, defined (see [Bou]) by

$$
d_{x}(\xi, \eta)=\lim _{t \rightarrow+\infty} e^{\frac{1}{2}\left(d\left(\xi_{t}, \eta_{t}\right)-d\left(x, \xi_{t}\right)-d\left(x, \eta_{t}\right)\right)},
$$

where $t \mapsto \xi_{t}, \eta_{t}$ are any geodesic rays ending at $\xi, \eta$ respectively. We endow from now on $\partial_{\infty} \widetilde{M}$ with the distance $d_{x}$.

The aim of this section is to compute the Hausdorff dimension of the Patterson measure $\mu_{x}^{F}$ associated with the potential $F$ (which will be independent of $x$ ). Recall that the Hausdorff dimension $\operatorname{dim}_{H}(v)$ of a finite nonzero measure $v$ on a locally compact

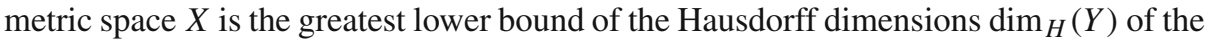
Borel subsets $Y$ of $X$ with $v(Y)>0$.

Let us give a motivation for such a computation. As mentioned in the introduction, we are interested in this paper in studying whether the set $E(\psi)$ of vectors of $T^{1} M$ that are well-spiraling, as quantified by $\psi$, around a given closed geodesic $D_{0}$ has full or zero measure for the Gibbs measure $m_{F}$. Varying the potential $F$ may be useful to estimate the 
Hausdorff dimension of $E(\psi)$ : if $\int_{0}^{+\infty} e^{\psi(t)\left(P\left(F_{\mid T^{1}} D_{0}\right)-P(F)\right)} d t$ diverges, as we will prove in Sect. 4, the set $E(\psi)$ has full measure for $m_{F}$, and hence $\operatorname{dim}_{H}(E(\psi)) \geq \operatorname{dim}_{H}\left(m_{F}\right)$. Note that the Hopf parametrisation of $T^{1} \widetilde{M}$ is Hölder-continuous (though usually not Lipschitz, except in particular when $\widetilde{M}$ is a symmetric space), and $\widetilde{m}_{F}$ is in the same measure class as the product measure $d \mu_{x}^{F \circ l} \otimes d \mu_{x}^{F} \otimes d t$. Hence $\operatorname{dim}_{H}\left(m_{F}\right)$ may be estimated using $\operatorname{dim}_{H}\left(\mu_{x}^{F}\right)$ (that we will prove to be equal to $\operatorname{dim}_{H}\left(\mu_{x}^{F \circ \iota}\right)$ ), and is in fact equal to $2 \operatorname{dim}_{H}\left(\mu_{x}^{F}\right)+1$ if $\widetilde{M}$ is a symmetric space.

The main result of this section, proving Theorem 1.1 in the introduction, is the following one. To simplify the notation, let $h(m)=h_{\frac{m}{\|m\|}}\left(g^{1}\right)$ be the (metric) entropy of

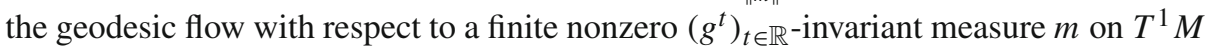
normalised to be a probability measure.

Theorem 3.1. If the Gibbs measure $m_{F}$ is finite and if $F$ is $m_{F}$-integrable, then the Hausdorf dimension of the Patterson measure $\mu_{x}^{F}$ on $\left(\partial_{\infty} \widetilde{M}, d_{x}\right)$ associated with $F$ satisfies

$$
\operatorname{dim}_{H}\left(\mu_{x}^{F}\right)=\operatorname{dim}_{H}\left(\mu_{x}^{F \circ \iota}\right)=h\left(m_{F}\right) \leq \delta_{\Gamma} .
$$

If $M$ is convex-cocompact, then the last inequality is an equality if and only if $F-P(F)$ is cohomologous to the zero potential.

We think that the convex-cocompact assumption in the last claim could be improved (see the comment at the end of this section).

The first claim is a generalisation of a result of Ledrappier [Led4], who proved the theorem in the particular case $F=0$. Then $\mu_{x}^{0}$ is the standard Patterson measure of $\Gamma$ and the associated Gibbs measure $m_{F}$ is the Bowen-Margulis measure $m_{\mathrm{BM}}$. Let $\Lambda_{c} \Gamma$ denote the conical (or radial) limit set, that is, the set of $\xi \in \partial_{\infty} \widetilde{M}$ for which $\liminf _{t \rightarrow+\infty} d(\rho(t), \Gamma x)<+\infty$, where $\rho$ is any geodesic ray with point at infinity $\xi$. Let $h_{\text {top }}\left(g^{1}\right)$ be the topological entropy of the geodesic flow on $T^{1} M$. If $m_{\mathrm{BM}}$ is finite, then Ledrappier [Led4, Theo. 4.3] proves furthermore that

$$
\operatorname{dim}_{H}\left(\mu_{x}^{0}\right)=h\left(m_{\mathrm{BM}}\right)=h_{\mathrm{top}}\left(g^{1}\right)=\operatorname{dim}_{H}\left(\Lambda_{c} \Gamma\right)=\delta_{\Gamma} .
$$

The second equality is due to Otal and Peigné [OP]. The last equality, which does not require the assumption that $m_{\mathrm{BM}}$ is finite, is due to Bishop-Jones in constant curvature, to Hamenstädt and to the first author (see [Pau]) in general.

Proof. Up to normalising $\mu_{x}^{F}$, which does not change its Hausdorff dimension nor $\frac{m_{F}}{\left\|m_{F}\right\|}$, we may assume that $\mu_{x}^{F}$ is a probability measure. The proof will follow from a series of claims. The following is a well known useful alternative characterisation of the dimension of the measure, which was also used by Ledrappier [Led4, Prop. 2.5].

Lemma 3.2. For any finite nonzero measure $v$ on a compact metric space $X$, the Hausdorff dimension $\operatorname{dim}_{H}(v)$ is the $v$-essential greatest lower bound on $x^{\prime} \in X$ of

$$
\liminf _{\epsilon \rightarrow 0} \frac{\log v\left(B\left(x^{\prime}, \epsilon\right)\right)}{\log \epsilon} .
$$

For every $\xi \in \partial_{\infty} \widetilde{M}$, let $\rho_{\xi}:\left[0,+\infty\left[\rightarrow \widetilde{M}\right.\right.$ be the geodesic ray with $\rho_{\xi}(0)=x$ and $\rho_{\xi}(+\infty)=\xi$. The next lemma compares shadows of balls in $\widetilde{M}$ with (visual) balls in $\partial_{\infty} \widetilde{M}$. 
Lemma 3.3 (Bourdon [Bou]). For sufficiently large $R>0$, there exists $D=D(R)$ such that, for all $\epsilon>0$ and $\xi \in \partial_{\infty} \widetilde{M}$,

$$
\mathscr{O}_{x}\left(B\left(\rho_{\xi}(\log (1 / \epsilon)+D), R\right)\right) \subset B_{d_{x}}(\xi, \epsilon) \subset \mathscr{O}_{x}\left(B\left(\rho_{\xi}(\log (1 / \epsilon)-D), R\right)\right) .
$$

Our first step in proving the theorem is the following result.

Proposition 3.4.(1) If $(\Gamma, F)$ is of divergence type then $\operatorname{dim}_{H}\left(\mu_{x}^{F}\right) \leq \operatorname{dim}_{H}\left(\Lambda_{c} \Gamma\right)=$ $\delta_{\Gamma} ;$

(2) If $m_{F}$ is finite and if $F$ is $m_{F}$-integrable, then

$$
\operatorname{dim}_{H}\left(\mu_{x}^{F}\right) \leq P(F)-\frac{1}{\left\|m_{F}\right\|} \int_{T^{1} M} F d m_{F} .
$$

Proof. By [PPS, Theo. 5.12], if $(\Gamma, F)$ is of divergence type, then the set $\Lambda_{c} \Gamma$ has full $\mu_{x}^{F}$-measure, and thus the inequality in Part (1) follows immediately from the definition of the Hausdorff dimension of measures. The equality in Part (1) has already been mentioned.

In order to prove Part (2), note that $(\Gamma, F)$ is of divergence type if $m_{F}$ is finite, by [PPS, Coro. 5.15]. It hence suffices by Lemma 3.2 to show that for $\mu_{x}^{F}$-almost every $\xi$ in the full $\mu_{x}^{F}$-measure subset $\Lambda_{c} \Gamma$, we have

$$
\liminf _{\epsilon \rightarrow 0} \frac{\log \mu_{x}^{F}\left(B_{d_{x}}(\xi, \epsilon)\right)}{\log \epsilon} \leq P(F)-\frac{1}{\left\|m_{F}\right\|} \int_{T^{1} M} F d m_{F} .
$$

Let $\xi \in \Lambda_{c} \Gamma$. By the definition of $\Lambda_{c} \Gamma$, there exist $K \geq 0$, a sequence $\left(\gamma_{n}\right)_{n \in \mathbb{N}}$ in $\Gamma$ and a sequence $\left(t_{n}\right)_{n \in \mathbb{N}}$ converging to $+\infty$ in $\left[0,+\infty\left[\right.\right.$ such that $d\left(\rho_{\xi}\left(t_{n}\right), \gamma_{n} x\right) \leq K$. By the triangle inequality, we have $d\left(x, \gamma_{n} x\right) \leq t_{n}+K$ and the ball $B\left(\rho_{\xi}\left(t_{n}\right), R\right)$ contains the ball $B\left(\gamma_{n} x, R-K\right)$, for every $R \geq K$. Let us apply the inclusion on the left in Lemma 3.3 with $\epsilon_{n}=e^{-t_{n}+D(R)}$, which tends to 0 as $n \rightarrow+\infty$ (hence in particular may be assumed to be in $] 0,1]$ ). We have

$$
\frac{\log \mu_{x}^{F}\left(B_{d_{x}}\left(\xi, \epsilon_{n}\right)\right)}{\log \epsilon_{n}} \leq \frac{\log \mu_{x}^{F}\left(\mathscr{O}_{x}\left(B\left(\rho_{\xi}\left(t_{n}\right), R\right)\right)\right)}{\log \epsilon_{n}} \leq \frac{\log \mu_{x}^{F}\left(\mathscr{O}_{x}\left(B\left(\gamma_{n} x, R-K\right)\right)\right)}{\log \epsilon_{n}}
$$

By Mohsen's shadow lemma (see Eq. (8)) and by [PPS, Theo. 6.1] which says that $P(F)=\delta_{\Gamma, F}$, if $R$ is large enough, there exists $c>0$ such that, for every $n \in \mathbb{N}$,

$$
\mu_{x}^{F}\left(\mathscr{O}_{x}\left(B\left(\gamma_{n} x, R-K\right)\right)\right) \geq \frac{1}{c} e^{\int_{x}^{\gamma_{n} x}(\widetilde{F}-P(F))}
$$

By Eq. (5), we have $\int_{x}^{\gamma_{n} x} \widetilde{F}=\int_{0}^{t_{n}} \widetilde{F}\left(\dot{\rho}_{\xi}(s)\right) d s+\mathrm{O}(1)$ as $n \rightarrow+\infty$. Thus Eq. (11) gives, as $n \rightarrow+\infty$,

$$
\begin{aligned}
\frac{\log \mu_{x}^{F}\left(B_{d_{x}}\left(\xi, \epsilon_{n}\right)\right)}{\log \epsilon_{n}} & \leq \frac{\int_{x}^{\gamma_{n} x}(\widetilde{F}-P(F))-\log c}{-t_{n}+D(R)} \\
& \leq\left(P(F)-\frac{1}{t_{n}} \int_{0}^{t_{n}} \widetilde{F}\left(\dot{\rho}_{\xi}(s)\right) d s\right)(1+\mathrm{o}(1))
\end{aligned}
$$


By [PPS, Theo. 5.4], since $(\Gamma, F)$ is of divergence type, the geodesic flow in $T^{1} M$ is ergodic for $m_{F}$. Since $F$ is $m_{F}$-integrable on $T^{1} M$, since $m_{F}$ is finite and by the quasiproduct structure of $\widetilde{m}_{F}$ in Hopf's parametrisation, for $\mu_{x}^{F}$-almost every $\xi$, we have by Birkhoff's ergodic theorem

$$
\lim _{n \rightarrow+\infty} \frac{1}{t_{n}} \int_{0}^{t_{n}} \tilde{F}\left(g^{s} \dot{\rho}_{\xi}(0)\right) d s=\frac{1}{\left\|m_{F}\right\|} \int_{T^{1} M} F d m_{F} .
$$

This proves Proposition 3.4.

We next want to show that the reverse inequality holds.

Proposition 3.5. If $m_{F}$ is a finite measure and if $F$ is $m_{F}$-integrable, then $\operatorname{dim}_{H}\left(\mu_{x}^{F}\right) \geq$ $P(F)-\frac{1}{\left\|m_{F}\right\|} \int_{T^{1} M} F d m_{F}$.

Proof. To prove the result, by Proposition 3.2, we only need to show that for $\mu_{x}^{F}$-almost every $\xi$, we have

$$
\liminf _{\epsilon \rightarrow 0} \frac{\log \mu_{x}^{F}\left(B_{d_{x}}(\xi, \epsilon)\right)}{\log \epsilon} \geq P(F)-\frac{1}{\left\|m_{F}\right\|} \int_{T^{1} M} F d m_{F} .
$$

As in the proof of [Led4, Prop. 4.6], since $m_{F}$ is finite and by the quasi-product structure of $\widetilde{m}_{F}$, by Poincaré's recurrence theorem and Birkhoff's ergodic theorem, for $\mu_{x}^{F}$-almost every $\xi$, there exist $K>0$, a sequence $\left(\gamma_{n}\right)_{n \in \mathbb{N}}$ in $\Gamma$ and an increasing sequence $\left(t_{n}\right)_{n \in \mathbb{N}}$, converging to $+\infty$ in $\left[0,+\infty\left[\right.\right.$, such that $d\left(\rho_{\xi}\left(t_{n}\right), \gamma_{n} x\right) \leq K$, and

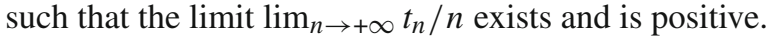

Let $R$ be big enough and let $c=c(R+K)$ be as in Mohsen's shadow lemma (see Eq. (8)), so that, for every $n \in \mathbb{N}$,

$$
\mu_{x}^{F}\left(\mathscr{O}_{x}\left(B\left(\gamma_{n} x, R+K\right)\right)\right) \leq c e^{\int_{x}^{\gamma_{n} x}(F-P(F))} .
$$

By the triangle inequality, the ball $B\left(\gamma_{n} x, R+K\right)$ contains the ball $B\left(\rho_{\xi}\left(t_{n}\right), R\right)$. For every $n \in \mathbb{N}$, let $\epsilon_{n}=e^{-t_{n}-D(R)}$, which decreases to 0 . For every $\left.\epsilon \in\right] 0$, 1] small enough, let $n=n(\epsilon) \in \mathbb{N}$ be such that $\epsilon_{n} \geq \epsilon>\epsilon_{n+1}$. By the inclusion on the right in Lemma 3.3 and by the same arguments as in the end of the proof of the previous proposition, we have

$$
\begin{aligned}
\frac{\log \mu_{x}^{F}\left(B_{d_{x}}(\xi, \epsilon)\right)}{\log \epsilon} & \geq \frac{\log \mu_{x}^{F}\left(B_{d_{x}}\left(\xi, \epsilon_{n}\right)\right)}{\log \epsilon_{n+1}} \geq \frac{\log \mu_{x}^{F}\left(\mathscr{O}_{x}\left(B\left(\rho_{\xi}\left(t_{n}\right), R\right)\right)\right)}{\log \epsilon_{n+1}} \\
& =\frac{-\log \mu_{x}^{F}\left(\mathscr{O}_{x}\left(B\left(\rho_{\xi}\left(t_{n}\right), R\right)\right)\right)}{t_{n+1}+D(R)} \geq \frac{-\log \mu_{x}^{F}\left(\mathscr{O}_{x}\left(B\left(\gamma_{n} x, R+K\right)\right)\right)}{t_{n+1}+D(R)} \\
& \geq \frac{-\int_{x}^{\gamma_{n} x}(F-P(F))-\log c}{t_{n+1}+D(R)} \\
& \geq \frac{t_{n} P(F)-\frac{t_{n}+\mathrm{o}\left(t_{n}\right)}{\left\|m_{F}\right\|} \int_{T^{1} M} F d m_{F}+\mathrm{O}(1)}{t_{n+1}+D(R)} .
\end{aligned}
$$

Taking the inferior limit as $\epsilon \rightarrow 0$, since $\lim _{n \rightarrow+\infty} \frac{t_{n}}{t_{n+1}}=1$, the result follows.

Now, by the Variational Principle [PPS, Theo. 6.1], since $m_{F}$ is finite and since $F$ is $m_{F}$-integrable, we have $P(F)=h\left(m_{F}\right)+\frac{1}{\left\|m_{F}\right\|} \int_{T^{1} M} F d m_{F}$. Since $\iota: T^{1} M \rightarrow T^{1} M$ 
conjugates $\left(g^{t}\right)_{t \in \mathbb{R}}$ to $\left(g^{-t}\right)_{t \in \mathbb{R}}$, and since $m_{F \circ \iota}=\iota_{*} m_{F}$, we have $h\left(m_{F \circ \iota}\right)=h\left(m_{F}\right)$. Hence Eq. (10) in Theorem 3.1 follows from Propositions 3.4 and 3.5 applied to both $F$ and $F \circ \iota$.

If $\Gamma$ is convex-cocompact, then $m_{F}$ and $m_{\mathrm{BM}}=m_{0}$ are finite and $F$ is integrable for $m_{F}$ and $m_{0}$. By the uniqueness in the Variational Principle (see [PPS, Theo. 6.1]), if $h\left(m_{F}\right)=\delta_{\Gamma}=h\left(m_{0}\right)$, then $\frac{m_{F}}{\left\|m_{F}\right\|}=\frac{m_{0}}{\left\|m_{0}\right\|}$. By the Hamenstädt-Ledrappier correspondence (see [Led3, Ham2, Sch] and the following proposition) saying that if $\Gamma$ is convex-cocompact, the cohomology class of a potential with zero pressure is determined by its associated Gibbs measure, the last claim of Theorem 3.1 follows.

We end this section by a comment on the correspondence between the potentials and their associated Patterson measures, which will be used at the end of this paper.

Proposition 3.6 (Hamenstädt-Ledrappier). If $\Gamma$ is convex-cocompact, the map $\widetilde{F} \mapsto$ $\mu=\mu_{x_{0}}^{F}$ induces a bijection from the set of $\Gamma$-invariant Hölder maps $\widetilde{F}: \widetilde{\Omega} \Gamma \rightarrow$ $\mathbb{R}$ with zero pressure $P(F)=0$, up to cohomologous maps, to the set of measure classes of Hölder quasi-invariant measures $\mu$ on $\left(\Lambda \Gamma, d_{x}\right)$ endowed with the action of $\Gamma$. Furthermore, for every hyperbolic element $\gamma \in \Gamma$ with attractive fixed point $\gamma^{+} \in \Lambda \Gamma$, the period of $\gamma$ for $\widetilde{F}$ satisfies

$$
\operatorname{Per}_{F}(\gamma)=\log \frac{d\left(\gamma^{-1}\right)_{*} \mu}{d \mu}\left(\gamma^{+}\right) .
$$

Proof. The reader who is not interested in seeing how this result can be deduced from [Led3] (whose arguments extend from the cocompact to the convex-cocompact case, as observed in [Sch]) may skip this proof.

Recall that $\partial_{\infty} \widetilde{M}$ has a unique Hölder structure such that for every $x \in \widetilde{M}$, the map $v \mapsto v_{+}$from $T_{x}^{1} \widetilde{M}$ to $\partial_{\infty} \widetilde{M}$ (whose inverse will be denoted by $\xi \mapsto v_{x}, \xi$ ) is a Hölder homeomorphism.

The following definitions are taken from [Led3]. A Hölder cocycle for the action of $\Gamma$ on $\partial_{\infty} \tilde{M}$ is a map $c: \Gamma \times \Lambda \Gamma \rightarrow \mathbb{R}$, which is Hölder-continuous in the second variable, such that $c\left(\gamma \gamma^{\prime}, \xi\right)=c\left(\gamma, \gamma^{\prime} \xi\right)+c\left(\gamma^{\prime}, \xi\right)$ for all $\gamma, \gamma^{\prime} \in \Gamma$ and $\xi \in \Lambda \Gamma$. The period for $c$ of a hyperbolic element $\gamma$ of $\Gamma$ is $c\left(\gamma, \gamma^{+}\right)$, where $\gamma^{+}$is the attractive fixed point of $\gamma$. Two Hölder cocycles $c$ and $c^{\prime}$ are cohomologous if there exists a Hölder-continuous map $U: \Lambda \Gamma \rightarrow \mathbb{R}$ such that $c(\gamma, \xi)-c^{\prime}(\gamma, \xi)=U(\gamma \xi)-U(\xi)$ for all $\gamma \in \Gamma$ and $\xi \in \Lambda \Gamma$. Given a Hölder quasi-invariant measure $\mu$, its associated Hölder cocycle is $c_{\mu}:(\gamma, \xi) \mapsto-\log \frac{d\left(\gamma^{-1}\right)_{*} \mu}{d \mu}(\xi)$. The verification that this is indeed a Hölder cocycle is immediate.

Fix $x_{0} \in \tilde{\widetilde{\Omega}} \Gamma$. Given a potential (that is, a $\Gamma$-invariant Hölder map) $\widetilde{F}: \widetilde{\Omega} \Gamma \rightarrow \mathbb{R}$, the map $c_{F}:(\gamma, \xi) \mapsto C_{\xi}^{F}\left(\gamma^{-1} x_{0}, x_{0}\right)$ is a Hölder cocycle (see [PPS, Prop. 3.5 (ii)] for its Hölder-continuity, $\widetilde{F}$ being bounded since $\Gamma \backslash \widetilde{\Omega} \Gamma$ is compact). Hence, by the definition of a Patterson density, given a potential $\widetilde{F}: \widetilde{\Omega} \Gamma \rightarrow \mathbb{R}$, the measure $\mu_{x_{0}}^{F}$ is a Hölder quasiinvariant measure, whose associated Hölder cocycle is $c_{F}$. If two potentials $\widetilde{F}$ and $\widetilde{F}^{*}$ are cohomologous, then their associated Hölder cocycles $c_{F}$ and $c_{F^{*}}$ are cohomologous: it is easy to check that if $\widetilde{G}: \widetilde{\Omega} \Gamma \rightarrow \mathbb{R}$ is Hölder-continuous, $\Gamma$-invariant, differentiable along every flow line, and satisfies Eq. (3), then the map $U: \Lambda \Gamma \rightarrow \mathbb{R}$ defined by $\xi \mapsto \widetilde{G}\left(v_{x_{0}}, \xi\right)$ is Hölder-continuous and satisfies $c_{F^{*}}(\gamma, \xi)-c_{F}(\gamma, \xi)=U(\gamma \xi)-U(\xi)$ for all $\gamma \in \Gamma$ and $\xi \in \Lambda \Gamma$. 
Let us relate the periods of a potential $F$ to the periods of the Hölder cocycle $c_{F}$. Let $\gamma$ be a hyperbolic element of $\Gamma$, with translation axis $A_{\gamma}$, translation length $\ell(\gamma)$ and attractive fixed point $\gamma_{+}$. By the $\Gamma$-invariance and the cocycle property of $C^{F}$, if $p$ is the closest point to $x_{0}$ on $A_{\gamma}$, we have $C_{\gamma^{+}}^{F}\left(\gamma^{-1} x_{0}, x_{0}\right)=C_{\gamma^{+}}^{F}\left(\gamma^{-1} p, p\right)$. Hence, by the definition of $C^{F}$, with $t \mapsto \xi_{t}$ the geodesic ray from $p$ to $\gamma^{+}$, we have (note that there are sign differences with [Led3])

$$
\begin{aligned}
c_{F}\left(\gamma, \gamma^{+}\right) & =C_{\gamma^{+}}^{F}\left(\gamma^{-1} x_{0}, x_{0}\right)=C_{\gamma^{+}}^{F}\left(\gamma^{-1} p, p\right) \\
& =\lim _{t \rightarrow+\infty} \int_{p}^{\xi_{t}}(\widetilde{F}-P(F))-\int_{\gamma^{-1} p}^{t}(\widetilde{F}-P(F)) \\
& =-\int_{\gamma^{-1} p}^{p}(\widetilde{F}-P(F))=P(F) \ell(\gamma)-\operatorname{Per}_{F}(\gamma) .
\end{aligned}
$$

By [Led3, Théo. 1.c], two Hölder quasi-invariant measures have the same measure class if and only if their associated Hölder cocycles are cohomologous, and this holds if and only if the periods of these Hölder cocycles are the same. By Livšic's theorem (see [PPS, Rem. 3.1]), two potentials $\widetilde{F}$ and $\widetilde{F}^{*}$ are cohomologous if and only they have the same periods. By Eq. (13), the periods of two potentials $\widetilde{F}$ and $\widetilde{F}^{*}$ with zero pressure are the same if and only if the periods of the associated Hölder cocycles $c_{F}$ and $c_{F^{*}}$ are the same. Hence the map which associates to the cohomology class of a potential $\widetilde{F}$ the measure class of the Hölder quasi-invariant measure $\mu_{x_{0}}^{F}$ is well-defined, and is injective. To prove that it is surjective, we start with a Hölder quasi-invariant measure $\mu$, we consider its associated Hölder cocycle $c_{\mu}$, the proof of [Led3, Théo. 3] shows that there exists a potential $\widetilde{F}$ such that the Hölder cocycle $c_{F}$ is cohomologous to $c_{\mu}$, and we apply again [Led3, Théo. 1.c] to get that $\mu_{x_{0}}^{F}$ and $\mu$ have the same measure class.

In order to prove Eq. (12), if $\widetilde{F}$ is a potential with $P(F)=0$, we have, by Eq. (13),

$$
\log \frac{d\left(\gamma^{-1}\right)_{*} \mu_{x_{0}}^{F}}{d \mu_{x_{0}}^{F}}\left(\gamma^{+}\right)=-c_{F}\left(\gamma, \gamma^{+}\right)=\operatorname{Per}_{F}(\gamma) .
$$

It would be interesting to know if one could remove the assumption that $\Gamma$ is convexcocompact, up to adding the requirements on $\widetilde{F}$ that $\delta_{\Gamma, F}$ is finite and $(\Gamma, F)$ is of divergence type, and on $\mu$ that $\mu$ is ergodic. This would improve correspondingly the last claim of Theorem 3.1 and simplify the statement of the requirement on the class of measures under consideration in Theorem 5.1.

\section{Almost Sure Spiraling for Gibbs States}

We will study in this section the generic asymptotic penetration properties of the geodesic lines, in a negatively curved simply connected manifold, under a discrete group of isometries, inside a tubular neighbourhood of a convex subset with cocompact stabiliser, not only as in [HP2] for the Bowen-Margulis measure, but for any Gibbs measure.

Let $\left(\widetilde{M}, \Gamma, \widetilde{F},\left(\mu_{x}^{F \circ \iota}\right)_{x \in \tilde{M}},\left(\mu_{x}^{F}\right)_{x \in \widetilde{M}}, \widetilde{m}_{F}\right)$ be as in the beginning of Sect. 3, with $\delta=\delta_{\Gamma, F}$ finite. We again use the notation introduced in Sect. 2.

Recall that a subgroup $H$ of a group $G$ is almost malnormal if, for every $g$ in $G-H$, the subgroup $g \mathrm{Hg}^{-1} \cap \mathrm{H}$ is finite. Let $\Gamma_{0}$ be an almost malnormal and convex-cocompact subgroup of $\Gamma$, of infinite index in $\Gamma$, let $C_{0}=\mathscr{C} \Lambda \Gamma_{0}$ be the convex hull of the limit set of $\Gamma_{0}$. For instance, $C_{0}$ could be the translation axis of a loxodromic element of $\Gamma$, and 
$\Gamma_{0}$ the stabiliser of $C_{0}$ in $\Gamma$ (see $[\mathrm{HP} 2, \S 4]$ for an explanation and for more examples). Up to adding assumptions on the behaviour of the potential and on growth properties in cusp neighbourhoods (including a gap property for the pressures), our result should extend when $\Gamma_{0}$ is assumed to be only geometrically finite instead of convex-cocompact, or when $\Gamma_{0}$ is a bounded parabolic group (in which case $\Gamma_{0}$ is also malnormal with infinite index in $\Gamma$ ) and $C_{0}$ is a precisely invariant closed horoball centred at the singleton $\Lambda \Gamma_{0}$. We restrict to the above case for simplicity.

Let $F_{0}: \Gamma_{0} \backslash T^{1} \widetilde{M} \rightarrow \mathbb{R}$ be the map induced by $\widetilde{F}$, and let $\delta_{0}=\delta_{\Gamma_{0}}, F_{0}$ be the critical exponent of $\left(\Gamma_{0}, F_{0}\right)$. Note that $-\infty<\delta_{0} \leq \delta<+\infty$ by [HP2, Lem. 3.3 (iii)].

Let $\psi:\left[0,+\infty\left[\rightarrow\left[0,+\infty\left[\right.\right.\right.\right.$ be a measurable map, such that there exist $c_{2}, c_{3}>0$ such that for every $s, t \geq c_{2}$, if $s \leq t+c_{2}$, then $\psi(s) \leq \psi(t)+c_{3}$. Recall (see for instance $[\mathrm{HP} 1, \S 5])$ that this condition is for instance satisfied if $\psi$ is Hölder-continuous; it implies that $e^{\psi}$ is locally bounded, hence it is locally integrable; and for every $\alpha>0$, the series $\sum_{n \in \mathbb{N}} e^{\alpha \psi(n)}$ converges if and only if the integral $\int_{0}^{+\infty} e^{\alpha \psi(t)} d t$ converges. Note that the constants $c_{2}$ and $c_{3}$ are unchanged by replacing $\psi$ by $\psi+c$ for any $c \in \mathbb{R}$.

Fix $\epsilon_{0}>0$. With the terminology of [HP2], let $\widetilde{E}(\psi)$ be the set of $\left(\epsilon_{0}, \psi\right)$-Liouville vectors for $\left(\Gamma, \Gamma_{0}\right)$ in $T^{1} \widetilde{M}$, that is, the set of $v \in T^{1} \widetilde{M}$ such that there exist a sequence $\left(t_{n}\right)_{n \in \mathbb{N}}$ in $\left[0,+\infty\right.$ [ converging to $+\infty$ and a sequence $\left(\gamma_{n}\right)_{n \in \mathbb{N}}$ in $\Gamma$ such that for every $t \in\left[t_{n}, t_{n}+\psi\left(t_{n}\right)\right]$, we have $g_{v}(t) \in \gamma_{n} \mathscr{N}_{\epsilon_{0}} C_{0}$. Note that $\widetilde{E}(\psi)$ is invariant under the geodesic flow and under $\Gamma$.

If $E$ is a set and $f, g: E \rightarrow] 0,+\infty[$ are maps, we write $f \asymp g$ if there exists $c>0$ such that $\frac{1}{c} f \leq g \leq c f$. The aim of this section is to prove the following result.

Theorem 4.1. Assume that the measure $m_{F}$ is finite, and that there exists $\kappa>0$ such that $\sum_{\gamma \in \Gamma, t \leq d(x, \gamma y)<t+\kappa} e^{\int_{x}^{\gamma y} \widetilde{F}} \asymp e^{t \delta}$ and $\sum_{\alpha \in \Gamma_{0}, t \leq d(x, \alpha y)<t+\kappa} e^{\int_{x}^{\alpha y} \widetilde{F}} \asymp e^{\delta_{0} t}$. If $\int_{0}^{+\infty} e^{\psi(t)\left(\delta_{0}-\delta\right)} d t$ diverges (resp. converges) then $\widetilde{m}_{F}$-almost every (resp. no) point of $T^{1} \widetilde{M}$ belongs to $\widetilde{E}(\psi)$.

Remarks (1) If the length spectrum of $\Gamma$ is nonarithmetic, then as said in Sect. 2, the measure $m_{F}$ is mixing for the geodesic flow on $T^{1} M$, hence by [PPS, Coro. 9.7], we have $\sum_{\gamma \in \Gamma, d(x, \gamma y) \leq t} e^{\int_{x}^{\gamma y} \widetilde{F}} \sim c e^{\delta t}$ as $t \rightarrow+\infty$, for some $c>0$, a stronger requirement than the first asymptotic hypothesis. Similarly, if the length spectrum of $\Gamma_{0}$ is nonarithmetic (this implies that $\Gamma_{0}$ is nonelementary), then the Gibbs measure $m_{F_{0}}$ of $\left(\Gamma_{0}, F_{0}\right)$, being finite since $\Gamma_{0}$ is convex-cocompact, is mixing, and the second asymptotic hypothesis holds. The fact that the second asymptotic hypothesis holds when $\Gamma_{0}$ is elementary (that is, when $C_{0}$ is the translation axis of a loxodromic element of $\Gamma$ ) is given by [PPS, Lem. 3.3 (ix)].

(2) The above theorem implies Theorem 1.2 in the introduction. Indeed, $M$ being compact, the measure $m_{F}$ is finite and the length spectrum of $\Gamma$ is nonarithmetic. Hence the two asymptotic hypotheses of Theorem 4.1 (which, up to changing $\kappa>0$, does not depend on the choice of $x, y \in \widetilde{M}$ ) hold by the previous remark. Note that if $C_{0}$ is the translation axis of a loxodromic element of $\Gamma$, if $D_{0}$ is its image by $\widetilde{M} \rightarrow M$, then $\delta_{0}=P\left(F_{\mid T^{1} D_{0}}\right)$ by [PPS, Lem. 3.3 (ix)]. We have $\delta=P(F)$ by [PPS, Theo. 6.1]. Hence the conclusion of Theorem 4.1 does imply Theorem 1.2.

Proof of Theorem 4.1. Before starting this proof, let us give more informations on $\Gamma_{0}$. Recall that $C_{0}$ is a non-compact, closed convex subset of $\widetilde{M}$ such that:

(1) $C_{0}$ is $\Gamma_{0}$-invariant and $\Gamma_{0} \backslash C_{0}$ is compact; up to replacing $\Gamma_{0}$ by $\mathrm{Stab}_{\Gamma} C_{0}$, in which $\Gamma_{0}$ has finite index and which remains almost malnormal (see the characterisation 
[HP2, Prop. $2.6(3)]$ ), so that $\delta_{0}$ and the validity of the second asymptotic hypothesis of Theorem 4.1 are unchanged, we may and we will assume that $\Gamma_{0}=\operatorname{Stab}_{\Gamma} C_{0}$;

(2) by [HP2, Prop. $2.6(2),(4)]$, the limit set $\Lambda \Gamma_{0}$ is precisely invariant (that is, we have $\gamma \Lambda \Gamma_{0} \cap \Lambda \Gamma_{0}=\emptyset$ for every $\left.\gamma \in \Gamma-\Gamma_{0}\right)$, and there exists $\kappa_{0}>0$ such that for every $\gamma \in \Gamma-\Gamma_{0}$, the diameter of $\mathscr{N}_{\epsilon_{0}} C_{0} \cap \gamma \mathscr{N}_{\epsilon_{0}} C_{0}$ is at most $\kappa_{0}$.

Lemma 4.2. If $\Gamma_{0}$ is a convex-cocompact subgroup of $\Gamma$, then $\delta_{0}<\delta$.

Proof. Since the Gibbs measure $m_{F_{0}}$ is finite, by [PPS, Theo. 6.1], the probability measure $\frac{m_{F_{0}}}{\left\|m_{F_{0}}\right\|}$ is an equilibrium state for the potential $F_{0}$ on $\Gamma_{0} \backslash T^{1} \tilde{M}$, whose support is contained in the compact nonwandering set $\Omega \Gamma_{0}=\Gamma_{0} \backslash \widetilde{\Omega} \Gamma_{0}$ of the geodesic flow on $\Gamma_{0} \backslash T^{1} \widetilde{M}$. Since $\Gamma_{0}$ is malnormal in $\Gamma$, the canonical map $p: \Gamma_{0} \backslash T^{1} \widetilde{M} \rightarrow \Gamma \backslash T^{1} \widetilde{M}$, when restricted on the nonwandering sets, is a finite-to-one map, by the above property (2). Hence if for a contradiction $\delta_{0}=\delta$, then $p_{*}\left(\frac{m_{F_{0}}}{\left\|m_{F_{0}}\right\|}\right)$ is an equilibrium state for $F$ on $\Gamma \backslash T^{1} \tilde{M}$. But by [PPS, Theo. 6.1], this equilibrium state is unique, hence $p_{*}\left(\frac{m_{F_{0}}}{\left\|m_{F_{0}}\right\|}\right)=\frac{m_{F}}{\left\|m_{F}\right\|}$.

Since $\Gamma_{0}$ is convex-cocompact and has infinite index in $\Gamma$, its limit set $\Lambda \Gamma_{0}$ is a precisely invariant (by the above property (2)) nonempty closed subset with empty interior in $\Lambda \Gamma$. Hence $\Gamma \Lambda \Gamma_{0}$ is a proper subset of $\Lambda \Gamma$ by Baire's theorem. Therefore the support of $p_{*} m_{F_{0}}$, which is the image by $p$ of $\Gamma_{0} \backslash \widetilde{\Omega} \Gamma_{0}=\Gamma_{0} \backslash\left\{v \in T^{1} \widetilde{M}: v_{-}, v_{+} \in\right.$ $\left.\Lambda \Gamma_{0}\right\}$, is a proper subset of the support $\Gamma \backslash \widetilde{\Omega} \Gamma=\Gamma \backslash\left\{v \in T^{1} \widetilde{M}: v_{-}, v_{+} \in \Lambda \Gamma\right\}$ of $m_{F}$, a contradiction.

We start the proof of Theorem 4.1 by two reductions of the statement.

(i) Up to adding a big enough constant to $\widetilde{F}$, which does not change $\widetilde{m}_{F}$, nor $\delta_{0}-\delta$, nor the asymptotics of the series in the above statement, we assume that $\delta_{0}>0$. In particular, $\delta$ is finite and positive.

(ii) Let $x_{0} \in C_{0}$ be a basepoint. Let $R_{0}>0$ and let $\widetilde{U_{0}}=\pi^{-1}\left(B\left(x_{0}, R_{0}\right)\right)$ be the set of the unit tangent vectors in $T^{1} \widetilde{M}$ based at a point at distance less that $R_{0}$ of $x_{0}$. If $R_{0}$ is big enough, then $m_{F}\left(\widetilde{U_{0}}\right)>0$. Since $m_{F}$ is finite, it is ergodic under the action of the geodesic flow on $T^{1} M$ (see [PPS, Coro. 5.15]). Hence the result is equivalent to proving that, when $R_{0}$ is big enough, if $\int_{0}^{+\infty} e^{\psi(t)\left(\delta_{0}-\delta\right)} d t$ diverges (resp. converges) then $\widetilde{m}_{F}$-almost every (resp. no) point of $\widetilde{U_{0}}$ belongs to $\widetilde{E}(\psi) \cap \widetilde{U_{0}}$.

We now define the various subsets of $\widetilde{U_{0}}$ that we will study during the proof of Theorem 4.1.

Let $E_{0}$ be the set of $[\gamma] \in \Gamma / \Gamma_{0}$ such that $d\left(x_{0}, \gamma C_{0}\right) \leq R_{0}+\epsilon_{0}$. Since $\Gamma$ is discrete, and since $\Gamma_{0}$ acts cocompactly on $C_{0}$, only finitely many distinct images of $C_{0}$ under $\Gamma$ meet a given compact subset of $\widetilde{M}$. In particular, the set $E_{0}$ is finite.

Since $\Gamma_{0} \backslash C_{0}$ is compact, let $\Delta_{0}>0$ be such that the restriction to the ball $B\left(x_{0}, \Delta_{0}\right)$ of the canonical projection $C_{0} \rightarrow \Gamma_{0} \backslash C_{0}$ is onto. Choose and fix once and for all a representative $\gamma$ of $[\gamma] \in \Gamma / \Gamma_{0}-E_{0}$ such that if $p_{\gamma}$ is the closest point to $x_{0}$ on $\gamma C_{0}$, then $d\left(p_{\gamma}, \gamma x_{0}\right) \leq \Delta_{0}$. We will use this representative whenever a coset is considered. For every $[\gamma] \in \Gamma / \Gamma_{0}-E_{0}$, define

$$
D_{\gamma}=d\left(x_{0}, \gamma C_{0}\right)=d\left(x_{0}, p_{\gamma}\right)>0 .
$$

Remark 4.3. Note that by an argument similar to [HP2, Lem. 4.1], for every $\lambda \in \mathbb{R}$, there are only finitely many $[\gamma] \in \Gamma / \Gamma_{0}-E_{0}$ such that $D_{\gamma} \leq \lambda$. 
Lemma 4.4. Assume that there exists $\kappa>0$ such that

$$
\sum_{\gamma \in \Gamma, t \leq d\left(x_{0}, \gamma x_{0}\right)<t+\kappa} e^{\int_{x_{0}}^{\gamma x_{0}} \widetilde{F}} \asymp e^{\delta t} \text { and } \sum_{\alpha \in \Gamma_{0}, t \leq d\left(x_{0}, \alpha x_{0}\right)<t+\kappa} e^{\int_{x_{0}}^{\alpha x_{0}} \widetilde{F}} \asymp e^{\delta_{0} t} .
$$

Then there exists $\kappa^{\prime} \geq 1$ such that $\sum_{[\gamma] \in \Gamma / \Gamma_{0}, t \leq D_{\gamma}<t+\kappa^{\prime}} e^{\int_{x_{0}}^{\gamma x_{0}} \widetilde{F}} \asymp e^{\delta t}$.

Proof. We start by proving that there exist $c_{4}, c_{5}>0$ such that for every $([\gamma], \alpha) \in$ $\Gamma / \Gamma_{0} \times \Gamma_{0}$, we have

$$
\begin{gathered}
D_{\gamma} \leq d\left(x_{0}, \gamma x_{0}\right) \leq D_{\gamma}+\Delta_{0}, \\
d\left(x_{0}, \gamma x_{0}\right)+d\left(x_{0}, \alpha x_{0}\right)-c_{4} \leq d\left(x_{0}, \gamma \alpha x_{0}\right) \leq d\left(x_{0}, \gamma x_{0}\right)+d\left(x_{0}, \alpha x_{0}\right), \\
\left|\int_{x_{0}}^{\gamma \alpha x_{0}} \widetilde{F}-\int_{x_{0}}^{\gamma x_{0}} \widetilde{F}-\int_{x_{0}}^{\alpha x_{0}} \widetilde{F}\right| \leq c_{5} .
\end{gathered}
$$

Equation (14), as well as the inequality on the right hand side of Eq. (15), follow by the triangle inequality:

$$
D_{\gamma}=d\left(x_{0}, \gamma C_{0}\right) \leq d\left(x_{0}, \gamma x_{0}\right) \leq d\left(x_{0}, p_{\gamma}\right)+d\left(p_{\gamma}, \gamma x_{0}\right) \leq D_{\gamma}+\Delta_{0} .
$$

By the convexity of $\gamma C_{0}$, the angle at $p_{\gamma}$ of the geodesic segments $\left[p_{\gamma}, x_{0}\right]$ and $\left[p_{\gamma}, \gamma \alpha x_{0}\right]$ (if they are non-trivial) is at least $\frac{\pi}{2}$. By hyperbolicity, the point $p_{\gamma}$ is hence at distance at most $\log (1+\sqrt{2})$ from a point in $\left[x_{0}, \gamma \alpha x_{0}\right]$. Thus $\gamma x_{0}$ is at distance at most $\Delta_{0}+\log (1+\sqrt{2})$ from a point $u$ in $\left[x_{0}, \gamma \alpha x_{0}\right]$. By the triangle inequality, the inequality on the left hand side of Eq. (15) follows with $c_{4}=2\left(\Delta_{0}+\log (1+\sqrt{2})\right)$.

Let us apply Eq. (5) twice, with $x=u, y=\gamma x_{0}$ and with either $z=x_{0}$ or $z=\gamma \alpha x_{0}$. Since $d\left(\gamma x_{0}, u\right) \leq \Delta_{0}+\log (1+\sqrt{2})$, Eq. (16) follows with

$$
c_{5}=2\left(c_{1} e^{\Delta_{0}+\log (1+\sqrt{2})}+\left(\Delta_{0}+\log (1+\sqrt{2})\right) \underset{\pi^{-1}\left(B\left(x_{0}, \Delta_{0}+\log (1+\sqrt{2})\right)\right)}{\max }|\widetilde{F}|\right) .
$$

We are now going to use the following lemma.

Lemma 4.5 [HP1, Lem. 3.3]. For all $A, \delta_{0}, \delta>0$, there exists $N \in \mathbb{N}$ and $B>0$ such that for all sequences $\left(a_{k}\right)_{k \in \mathbb{N}}$ and $\left(b_{k}\right)_{k \in \mathbb{N}}$ such that $a_{n} \leq A e^{\delta n}, b_{n} \leq A e^{\delta_{0} n}$ and $\sum_{k=0}^{n} a_{k} b_{n-k} \geq \frac{1}{A} e^{\delta n}$ for every $n \in \mathbb{N}$ big enough, we have $\sum_{k=0}^{N} a_{n+k} \geq B e^{\delta n}$ for every $n \in \mathbb{N}$.

By the first asymptotic assumption in Lemma 4.4, there exists $c^{\prime}>0$ such that, for every $t \geq \kappa$,

$$
\sum_{\gamma^{\prime} \in \Gamma, t-\kappa \leq d\left(x_{0}, \gamma^{\prime} x_{0}\right)<t} e^{\int_{x_{0}}^{\gamma^{\prime} x_{0}} \widetilde{F}} \geq \frac{1}{c} e^{\delta t}
$$

We will use Lemma 4.5 by taking, for every $k \in \mathbb{N}$,

$$
a_{k}=\sum_{[\gamma] \in \Gamma / \Gamma_{0}, k \leq D_{\gamma}<k+1} e^{\int_{x_{0}}^{\gamma x_{0}} \widetilde{F}} \text { and } b_{k}=\sum_{\alpha \in \Gamma_{0}, k-\kappa-c_{4} \leq d\left(x_{0}, \alpha x_{0}\right)<k} e^{\int_{x_{0}}^{\alpha x_{0}} \widetilde{F}}
$$


By Eq. (14) and by the first asymptotic assumption in Lemma 4.4, there exists $c^{\prime}>0$ such that, for every $k \in \mathbb{N}$,

$$
a_{k} \leq \sum_{\gamma \in \Gamma, k \leq d\left(x_{0}, \gamma x_{0}\right)<k+1+\Delta_{0}} e^{\int_{x_{0}}^{\gamma x_{0}} \widetilde{F}} \leq c^{\prime} e^{\delta k} .
$$

By the second asymptotic assumption in Lemma 4.4, there exists $c^{\prime \prime}>0$ such that, for every $k \in \mathbb{N}$,

$$
b_{k} \leq c^{\prime \prime} e^{\delta_{0} k}
$$

Let $n \geq \kappa+c_{4}$ and $([\gamma], \alpha) \in \Gamma / \Gamma_{0} \times \Gamma_{0}$ satisfy $n-\kappa-c_{4} \leq d\left(x_{0}, \gamma^{\prime} x_{0}\right)<n-c_{4}$ where $\gamma^{\prime}=\gamma \alpha$. Let $k=\left\lfloor D_{\gamma}\right\rfloor$ be the integral part of $D_{\gamma}$. By Eq. (15), we hence have

$$
0 \leq k \leq D_{\gamma} \leq d\left(x_{0}, \gamma x_{0}\right) \leq d\left(x_{0}, \gamma \alpha x_{0}\right)-d\left(x_{0}, \alpha x_{0}\right)+c_{4} \leq n,
$$

and

$$
\begin{aligned}
n-\kappa-c_{4}-k & \leq d\left(x_{0}, \gamma \alpha x_{0}\right)-d\left(x_{0}, \gamma x_{0}\right) \leq d\left(x_{0}, \alpha x_{0}\right) \\
& \leq d\left(x_{0}, \gamma \alpha x_{0}\right)-d\left(x_{0}, \gamma x_{0}\right)+c_{4} \leq n-k
\end{aligned}
$$

Therefore, respectively by the definition of $a_{k}$ and $b_{n-k}$, and by Eq. (16), we have

$$
\begin{aligned}
& \sum_{k=0}^{n} a_{k} b_{n-k}=\sum_{k=0}^{n} \sum_{[\gamma] \in \Gamma / \Gamma_{0}, k \leq D_{\gamma}<k+1} \sum_{\alpha \in \Gamma_{0}, n-k-\kappa-c_{4} \leq d\left(x_{0}, \alpha x_{0}\right)<n-k} e^{\int_{x_{0}}^{\gamma x_{0}} \widetilde{F}} e^{\int_{x_{0}}^{\alpha x_{0}} \widetilde{F}} \\
& \geq e^{-c_{5}} \sum_{\gamma^{\prime} \in \Gamma, n-\kappa-c_{4} \leq d\left(x_{0}, \gamma^{\prime} x_{0}\right)<n-c_{4}} e^{\int_{x_{0} x_{0}}^{\gamma^{\prime} x_{0}} \widetilde{F}} \geq e^{-c_{5}} \frac{1}{c} e^{\delta\left(n-c_{4}\right)} \text {. }
\end{aligned}
$$

Applying Lemma 4.5 with $A=\max \left\{c^{\prime}, c^{\prime \prime}, c e^{c_{5}+\delta c_{4}}\right\}$ gives the lower bound required to prove Lemma 4.4. The upper bound follows from Eq. (17).

For every $r>0$ and $\beta \in \Gamma$, let

$$
A_{\beta}(r)=\left\{v \in \widetilde{U_{0}}: g_{v}\left(\left[0,+\infty[) \cap B\left(\beta x_{0}, r\right) \neq \emptyset\right\} .\right.\right.
$$

Let us fix a positive constant $c_{6} \geq \kappa$ (depending only on $\epsilon_{0}, \Delta_{0}, R_{0}, \kappa$ and $\psi$ ) to be made precise later on. For every $k \in \mathbb{N}$, define $I_{k}$ to be the set of $[\gamma] \in \Gamma / \Gamma_{0}$ such that $k \leq D_{\gamma}<k+1$, and let $J_{k}=J_{k}(\psi)$ be the set of pairs $([\gamma], \alpha) \in \Gamma / \Gamma_{0} \times \Gamma_{0}$ such that $k \leq D_{\gamma}<k+\kappa^{\prime}$ (where $\kappa^{\prime}$ is given by Lemma 4.4) and $\psi(k) \leq d\left(x_{0}, \alpha x_{0}\right)<\psi(k)+c_{6}$. For every $k \in \mathbb{N}$, let

$$
A_{k}(r, \psi)=\bigcup_{([\gamma], \alpha) \in J_{k}} A_{\gamma \alpha}(r) .
$$

These sets are related to the set $\widetilde{E}(\psi)$ that we want to study by the following result. Recall that if $\left(B_{k}\right)_{k \in \mathbb{N}}$ is a sequence of subsets of a given set, one defines $\lim \sup _{k} B_{k}=$ $\bigcap_{n \in \mathbb{N}} \bigcup_{k \geq n} B_{k}$. 
Proposition 4.6. If $r \geq \epsilon_{0}+\Delta_{0}$, there exist $c_{5}^{\prime}, c_{5}^{\prime \prime}>0$ such that, up to sets of $\widetilde{m}_{F}$-measure zero,

$$
\underset{k}{\lim \sup } A_{k}\left(r, \psi+c_{5}^{\prime \prime}\right) \subset \widetilde{E}(\psi) \cap \widetilde{U_{0}}
$$

and if $\psi(t) \geq c_{5}^{\prime}$ for $t$ big enough,

$$
\widetilde{E}(\psi) \cap \widetilde{U_{0}} \subset \limsup _{k} A_{k}\left(r, \psi-c_{5}^{\prime}\right)
$$

Proof. Let us first prove the second inclusion. Let $c_{0}=\epsilon_{0}+2 \operatorname{arsinh}\left(\operatorname{coth} \epsilon_{0}\right)$. Let $c_{0}^{\prime}=c_{3}\left\lceil\frac{2 R_{0}+c_{0}}{c_{2}}\right\rceil$, with $c_{2}, c_{3}$ the constants appearing in the assumption on $\psi$. Let $c_{5}^{\prime}=\epsilon_{0}+2 \Delta_{0}+R_{0}+c_{0}+c_{0}^{\prime}$. Assume that $\psi(t) \geq c_{5}^{\prime}$ for $t$ big enough.

Let $v \in \widetilde{E}(\psi) \cap \widetilde{U_{0}}$. For every $n \in \mathbb{N}$, there exist sequences $\left(t_{n}\right)_{n \in \mathbb{N}}$ in $[0,+\infty[$ converging to $+\infty$ and $\left(\left[\gamma_{n}\right]\right)_{n \in \mathbb{N}}$ in $\Gamma / \Gamma_{0}$ such that for every $t \in\left[t_{n}, t_{n}+\psi\left(t_{n}\right)\right]$, we have $g_{v}(t) \in \gamma_{n} \mathscr{N}_{\epsilon_{0}} C_{0}$. Let $n \in \mathbb{N}$. The geodesic line $g_{v}$ enters in $\gamma_{n} \mathscr{N}_{\epsilon_{0}} C_{0}$ at a time $t_{n}^{-}$at most $t_{n}$. Up to extracting a subsequence, we may assume, by Remark 4.3, that $\left[\gamma_{n}\right] \notin E_{0}$, so that $D_{\gamma_{n}}>R_{0}+\epsilon_{0}$ and $t_{n}^{-}>0$.

Let $k_{n}=\left\lfloor D_{\gamma_{n}}\right\rfloor$. Let us prove that $k_{n} \rightarrow+\infty$ as $n \rightarrow+\infty$, up to sets of $\tilde{m}_{F}$-measure zero of elements $v \in \widetilde{E}(\psi) \cap \widetilde{U_{0}}$. Otherwise, up to extracting a subsequence, $\left(\gamma_{n}\right)_{n \in \mathbb{N}}$ is constant by Remark 4.3. Hence $v_{+}$belongs to the set $\gamma_{0} \partial_{\infty} C_{0}$ of accumulation points of $\gamma_{0} C_{0}$ in $\partial_{\infty} \widetilde{M}$. By Lemma 2.1, the $\mu_{x_{0}}^{F}$-measure of $\partial_{\infty} C_{0}=\Lambda \Gamma_{0}$ is zero. Hence, since the action of $\Gamma$ preserves the sets of $\mu_{x_{0}}^{F}$-measure zero by the properties of the Patterson densities, we have $\mu_{x_{0}}^{F}\left(\bigcup_{\beta \in \Gamma} \beta \partial_{\infty} C_{0}\right)=0$. By the decomposition of $\tilde{m}_{F}$ in Hopf's parametrisation (see Eq. (7)), the $\widetilde{m}_{F}$-measure of the set of $v \in \widetilde{E}(\psi)$ such that $v_{+} \in \bigcup_{\beta \in \Gamma} \beta \partial_{\infty} C_{0}$ is zero. This proves the above claim.

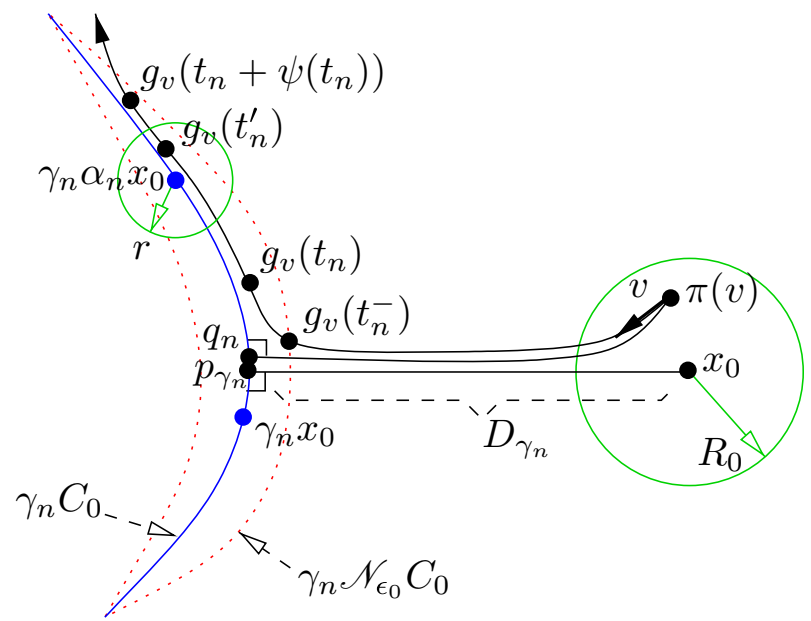

Let $q_{n}$ be the closest point to $\pi(v)$ on $\gamma_{n} C_{0}$. It satisfies $d\left(p_{\gamma_{n}}, q_{n}\right) \leq R_{0}$, since closest point maps do not increase the distances. Note that the point $q_{n}$ is at distance $\epsilon_{0}$ from the entry point in $\gamma_{n} \mathscr{N}_{\epsilon_{0}} C_{0}$ of the geodesic segment from $\pi(v)$ to $q_{n}$. By the penetration properties of geodesic rays in $\epsilon_{0}$-neighbourhoods of convex subsets of CAT $(-1)$ metric 
spaces (see [PaP1, Lem. 2.3]), we have $d\left(q_{n}, g_{v}\left(t_{n}^{-}\right)\right) \leq c_{0}=\epsilon_{0}+2 \operatorname{arsinh}\left(\operatorname{coth} \epsilon_{0}\right)$. Hence, by the triangle inequality,

$$
d\left(\gamma_{n} x_{0}, g_{v}\left(t_{n}^{-}\right)\right) \leq d\left(\gamma_{n} x_{0}, p_{\gamma_{n}}\right)+d\left(p_{\gamma_{n}}, q_{n}\right)+d\left(q_{n}, g_{v}\left(t_{n}^{-}\right)\right) \leq \Delta_{0}+R_{0}+c_{0} .
$$

Again by the triangle inequality, we have

$k_{n} \leq D_{\gamma_{n}}=d\left(x_{0}, p_{\gamma_{n}}\right) \leq d\left(x_{0}, \pi(v)\right)+t_{n}^{-}+d\left(g_{v}\left(t_{n}^{-}\right), q_{n}\right)+d\left(q_{n}, p_{\gamma_{n}}\right) \leq t_{n}+2 R_{0}+c_{0}$.

Up to extracting a subsequence, we may assume that $\psi\left(k_{n}\right) \geq c_{5}^{\prime}$ and that $t_{n}, k_{n} \geq c_{2}$. By the assumption on $\psi$ and since $c_{0}^{\prime}=c_{3}\left\lceil\frac{2 R_{0}+c_{0}}{c_{2}}\right\rceil$, we have by Eq. (19),

$$
t_{n}^{-}+\psi\left(k_{n}\right) \leq t_{n}+\psi\left(t_{n}\right)+c_{0}^{\prime}
$$

Let $t_{n}^{\prime}=t_{n}^{-}+\psi\left(k_{n}\right)-c_{0}^{\prime}$, which belongs to $\left[t_{n}^{-}, t_{n}+\psi\left(t_{n}\right)\right]$, since $\psi\left(k_{n}\right) \geq c_{5}^{\prime} \geq c_{0}^{\prime}$. By convexity, the point $g_{v}\left(t_{n}^{\prime}\right)$ belongs to $\mathscr{N}_{\epsilon_{0}} C_{0}$. Let $\alpha_{n}$ be an element of $\Gamma_{0}$ such that

$$
d\left(g_{v}\left(t_{n}^{\prime}\right), \gamma_{n} \alpha_{n} x_{0}\right) \leq \epsilon_{0}+\Delta_{0}
$$

which exists by the definition of $\Delta_{0}$. By the triangle inequality, and by Eq. (18), we have

$$
\begin{aligned}
\left|d\left(\gamma_{n} x_{0}, \gamma_{n} \alpha_{n} x_{0}\right)-d\left(g_{v}\left(t_{n}^{-}\right), g_{v}\left(t_{n}^{\prime}\right)\right)\right| & \leq d\left(g_{v}\left(t_{n}^{\prime}\right), \gamma_{n} \alpha_{n} x_{0}\right)+d\left(\gamma_{n} x_{0}, g_{v}\left(t_{n}^{-}\right)\right) \\
& \leq \epsilon_{0}+2 \Delta_{0}+R_{0}+c_{0} .
\end{aligned}
$$

Hence

$$
\begin{aligned}
\left|d\left(x_{0}, \alpha_{n} x_{0}\right)-\psi\left(k_{n}\right)\right| & =\left|d\left(\gamma_{n} x_{0}, \gamma_{n} \alpha_{n} x_{0}\right)-\right| t_{n}^{\prime}-t_{n}^{-}\left|-c_{0}^{\prime}\right| \\
& \leq \epsilon_{0}+2 \Delta_{0}+R_{0}+c_{0}+c_{0}^{\prime}=c_{5}^{\prime} .
\end{aligned}
$$

Define $c_{6}=\max \left\{2 c_{5}^{\prime}, \kappa\right\}$ (which only depends on $\epsilon_{0}, \Delta_{0}, R_{0}, \kappa$ and $\psi$ ). Assume that $r \geq \epsilon_{0}+\Delta_{0}$. For every $n \in \mathbb{N}$, we hence have $v \in A_{\gamma_{n} \alpha_{n}}(r)$ by Eq. (20). Besides, $\left(\left[\gamma_{n}\right], \alpha_{n}\right) \in J_{k_{n}}\left(\psi-c_{5}^{\prime}\right)$ since $k_{n}=\left\lfloor D_{\gamma_{n}}\right\rfloor$ and $\kappa^{\prime} \geq 1$, and by Eq. (21). Therefore $v \in A_{k_{n}}\left(r, \psi-c_{5}^{\prime}\right)$. This proves the second inclusion in Proposition 4.6.

Let us now prove the first inclusion. By hyperbolicity and an argument of (strict) convexity (see for instance [PaP1, Lem. 2.2]), there exists $c_{0}^{\prime \prime}=c_{0}^{\prime \prime}\left(\epsilon_{0}, R_{0}, r\right)$ such that if a geodesic segment has endpoints at distance at most $\max \left\{R_{0}, r\right\}+\log (1+\sqrt{2})$ from two points in $C_{0}$ at distance at least $c_{0}^{\prime \prime}$ one from the other, then this geodesic segment enters $\mathscr{N}_{\epsilon_{0}} C_{0}$. Let $c_{5}^{\prime \prime}=\max \left\{c_{0}^{\prime \prime}+\Delta_{0}, \Delta_{0}+R_{0}+2 c_{0}+r+c_{3}\left\lceil\frac{2 R_{0}+c_{0}+1}{c_{2}}\right\rceil\right\}$.

Let $v \in \widetilde{U}_{0}$ and let $\left(k_{n}\right)_{n \in \mathbb{N}}$ be a sequence in $\mathbb{N}$ converging to $+\infty$. Assume that $v \in$ $A_{k_{n}}\left(r, \psi+c_{5}^{\prime \prime}\right)$ for every $n$ in $\mathbb{N}$. Let $\left(\left[\gamma_{n}\right], \alpha_{n}\right) \in J_{k_{n}}\left(\psi+c_{5}^{\prime \prime}\right)$ be such that $v \in A_{\gamma_{n} \alpha_{n}}(r)$ : there exists $\tau_{n} \geq 0$ such that $g_{v}\left(\tau_{n}\right) \in B\left(\gamma_{n} \alpha_{n} x_{0}, r\right)$. Since $d\left(\pi(v), x_{0}\right) \leq R_{0}$, by the properties of closest point projections in CAT $(-1)$-space, there exists $\tau_{n}^{\prime} \in\left[0, \tau_{n}\right]$ such that $d\left(g_{v}\left(\tau_{n}^{\prime}\right), p_{\gamma_{n}}\right) \leq \max \left\{R_{0}, r\right\}+\log (1+\sqrt{2})$. By the definition of $c_{0}^{\prime \prime}$ and since 
$d\left(p_{\gamma_{n}}, \gamma_{n} \alpha_{n} x_{0}\right) \geq d\left(\gamma_{n} x_{0}, \gamma_{n} \alpha_{n} x_{0}\right)-\Delta_{0} \geq \psi\left(k_{n}\right)+c_{5}^{\prime \prime}-\Delta_{0} \geq c_{0}^{\prime \prime}$, the geodesic line $g_{v}$ enters $\gamma_{n} \mathscr{N}_{\epsilon_{0}} C_{0}$.

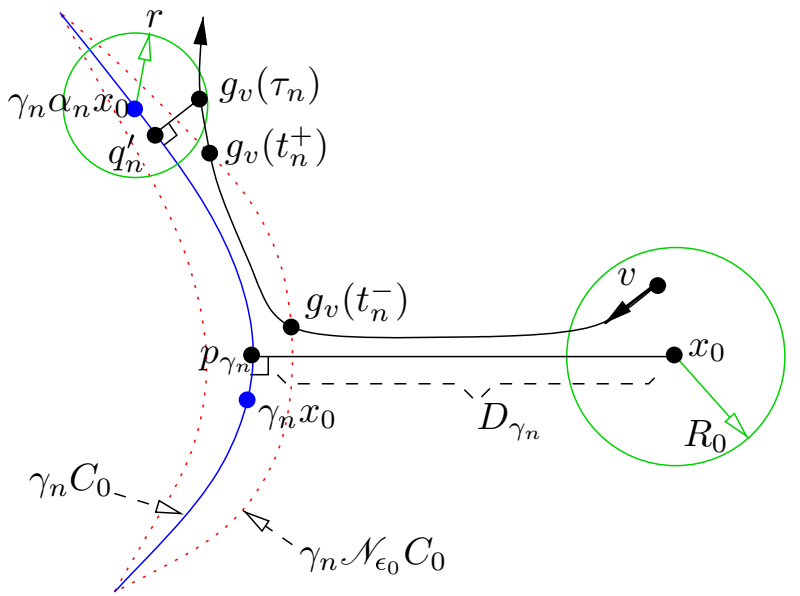

Let $t_{n}^{-}$be the entry time of $g_{v}$ inside $\gamma_{n} \mathscr{N}_{\epsilon_{0}} C_{0}$, which satisfies, by Eq. (18),

$$
d\left(g_{v}\left(t_{n}^{-}\right), \gamma_{n} x_{0}\right) \leq \Delta_{0}+R_{0}+c_{0} .
$$

Let $t_{n}^{+}$be either $\tau_{n}$ if $g_{v}\left(\tau_{n}\right) \in \gamma_{n} \mathscr{N}_{\epsilon_{0}} C_{0}$ or the exit time of $g_{v}$ out of $\mathscr{N}_{\epsilon_{0}} C_{0}$ otherwise. Again by [PaP1, Lem. 2.3] and since closest point maps do not increase the distances, if $q_{n}^{\prime}$ is the closest point to $g_{v}\left(\tau_{n}\right)$ on $C_{0}$, we have

$$
d\left(g_{v}\left(t_{n}^{+}\right), \gamma_{n} \alpha_{n} x_{0}\right) \leq d\left(g_{v}\left(t_{n}^{+}\right), q_{n}^{\prime}\right)+d\left(q_{n}^{\prime}, \gamma_{n} \alpha_{n} x_{0}\right) \leq c_{0}+r .
$$

As in Eq. (19), we have $k_{n} \geq D_{\gamma_{n}}-1 \geq t_{n}^{-}-2 R_{0}-c_{0}-1$. Hence

$$
\psi\left(k_{n}\right) \geq \psi\left(t_{n}^{-}\right)-c_{3}\left\lceil\frac{2 R_{0}+c_{0}+1}{c_{2}}\right\rceil .
$$

By the triangle inequality and since $\left(\left[\gamma_{n}\right], \alpha_{n}\right) \in J_{k_{n}}\left(\psi+c_{5}^{\prime \prime}\right)$, we have

$$
\begin{aligned}
t_{n}^{+}-t_{n}^{-} & \geq d\left(\gamma_{n} x_{0}, \gamma_{n} \alpha_{n} x_{0}\right)-d\left(g_{v}\left(t_{n}^{-}\right), \gamma_{n} x_{0}\right)-d\left(g_{v}\left(t_{n}^{+}\right), \gamma_{n} \alpha_{n} x_{0}\right) \\
& \geq \psi\left(k_{n}\right)+c_{5}^{\prime \prime}-\Delta_{0}-R_{0}-2 c_{0}-r \\
& \geq \psi\left(t_{n}^{-}\right) .
\end{aligned}
$$

Hence $v$ belongs to $\widetilde{E}(\psi)$, which proves the result.

In a series of claims, we now state the required properties of the sets $A_{\gamma \alpha}(r)$ for $([\gamma], \alpha) \in \Gamma / \Gamma_{0} \times \Gamma_{0}$ and $A_{k}(r, \psi)$ for $k \in \mathbb{N}$.

We start by the following estimate on the mass of the $A_{\gamma \alpha}(r)$ 's. Before stating it, let us motivate it. Let $d^{\prime}$ be the distance on $T^{1} \widetilde{M}$ induced by Sasaki's Riemannian metric on $T \widetilde{M}$ (when $\Gamma$ is cocompact, any Riemannian distance on $T^{1} \widetilde{M}$ is allowed). Recall that, for $\epsilon>0$ and $T \geq 0$, the dynamical $(\epsilon, T)$-ball centred at a point $v \in T^{1} \widetilde{M}$ is

$$
B_{\epsilon, T}(v)=\left\{w \in T^{1} \widetilde{M}: \forall t \in[0, T], \quad d^{\prime}\left(g^{t} w, g^{t} v\right) \leq \epsilon\right\} .
$$


We proved in [PPS, Prop. 3.16] (which was in fact written after the first version of this paper), using a minor modification of these dynamical balls, that Gibbs measures satisfy the Gibbs property (when $\Gamma$ is torsion free and cocompact, see for instance [BR, Theo. 3.3] for the lower bound, and [KH, Lem. 20.3.4] in the discrete time case): for every $\epsilon>0$, for all $v \in T^{1} \widetilde{M}$ and $T \geq 0$ such that $v, g^{T}(v)$ map to a given compact subset of $\Gamma \backslash T^{1} \widetilde{M}$, we have

$$
\widetilde{m}_{F}\left(B_{\epsilon, T}(v)\right) \asymp e^{\int_{0}^{T} \widetilde{F}\left(g^{t} v\right) d t-T P(F)} .
$$

Now, $A_{\gamma \alpha}(r)$ is almost such a dynamical ball. Indeed, let $v_{\gamma \alpha}$ be the unit tangent vector at $x_{0}$ of the geodesic segment from $x_{0}$ to $\gamma \alpha x_{0}$, and let $T_{\gamma \alpha}=d\left(x_{0}, \gamma \alpha x_{0}\right)$ (see the figure below). Our set $A_{\gamma \alpha}(r)$ contains $B_{\epsilon_{-}, T_{\gamma \alpha}}\left(v_{\gamma \alpha}\right)$ and is contained in $B_{\epsilon_{+}, T_{\gamma \alpha}}\left(v_{\gamma \alpha}\right)$ for some positive constants $\epsilon_{ \pm}$depending only on $R_{0}, r$. The following result (or rather Eq. (25)) is hence closely related to this Gibbs property.

Proposition 4.7. If $r$ and $R_{0}$ are big enough, there exists $c_{7}=c_{7}(r)>0$ such that for all but finitely many $([\gamma], \alpha) \in \Gamma / \Gamma_{0} \times \Gamma_{0}$, we have

$$
\frac{1}{c_{7}} e^{\int_{x_{0}}^{\gamma x_{0}}(\widetilde{F}-\delta)} e^{\int_{x_{0}}^{\alpha x_{0}}(\widetilde{F}-\delta)} \leq \widetilde{m}_{F}\left(A_{\gamma \alpha}(r)\right) \leq c_{7} e^{\int_{x_{0}}^{\gamma x_{0}}(\widetilde{F}-\delta)} e^{\int_{x_{0}}^{\alpha x_{0}}(\widetilde{F}-\delta)}
$$

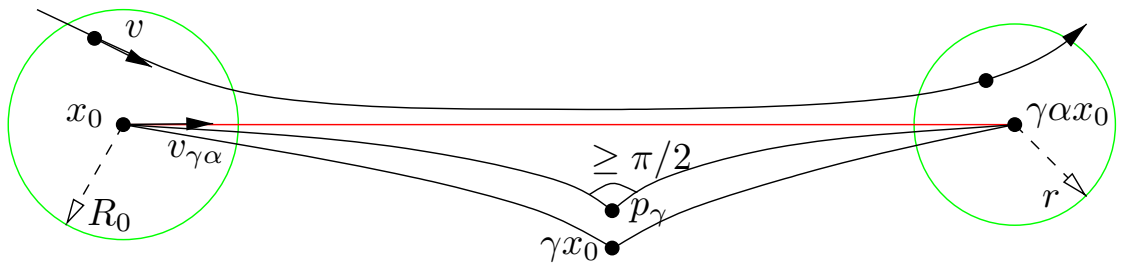

Proof. For every $R_{0}>0$ and $\beta \in \Gamma$, define $B_{R_{0}, \beta}=\bigcap_{z^{\prime} \in B\left(\beta x_{0}, r\right)} \mathscr{O}_{z^{\prime}} B\left(x_{0}, \frac{R_{0}}{3}\right)$, which is contained in (and is a perturbation of) the shadow $\mathscr{O}_{\beta x_{0}} B\left(x_{0}, \frac{R_{0}}{3}\right)$ (see the picture below). Since $\Gamma$ is nonelementary, the support of the Patterson measures is not reduced to one point, hence $m=\inf _{\xi \in \partial_{\infty} \widetilde{M}}\left\|\mu_{x_{0}}^{F \circ \iota}\right\|-\mu_{x_{0}}^{F \circ \iota}(\{\xi\})$ is positive. By hyperbolicity (as first remarked by Sullivan), for every $\xi \in \partial_{\infty} \widetilde{M}$, the family $\left({ }^{c} \mathscr{O}_{\xi} B\left(x_{0}, R\right)\right)_{R>0}$ is a fundamental system of neighbourhoods of $\xi$. By compactness and discreteness, there exists hence $R_{0}>0$ such that for all but finitely many $\beta \in \Gamma$, we have

$$
\mu_{x_{0}}^{F \circ \iota}\left(B_{R_{0}, \beta}\right) \geq \frac{m}{2}
$$

By the definition of $A_{\beta}(r)$, the set of points $v_{+}$for $v$ in $A_{\beta}(r)$ is exactly

$$
A_{\beta}(r)_{+}=\bigcup_{z \in B\left(x_{0}, R_{0}\right)} \mathscr{O}_{z} B\left(\beta x_{0}, r\right),
$$

which is a bit larger than the shadow $\mathscr{O}_{x_{0}} B\left(\beta x_{0}, r\right)$ (see the picture below). By a minor modification of Mohsen's shadow lemma (see Eq. (8)), we have, if $r$ is big enough,

$$
\mu_{x_{0}}^{F}\left(A_{\beta}(r)_{+}\right) \asymp e^{\int_{x_{0}}^{\beta x_{0}}(\widetilde{F}-\delta)} .
$$




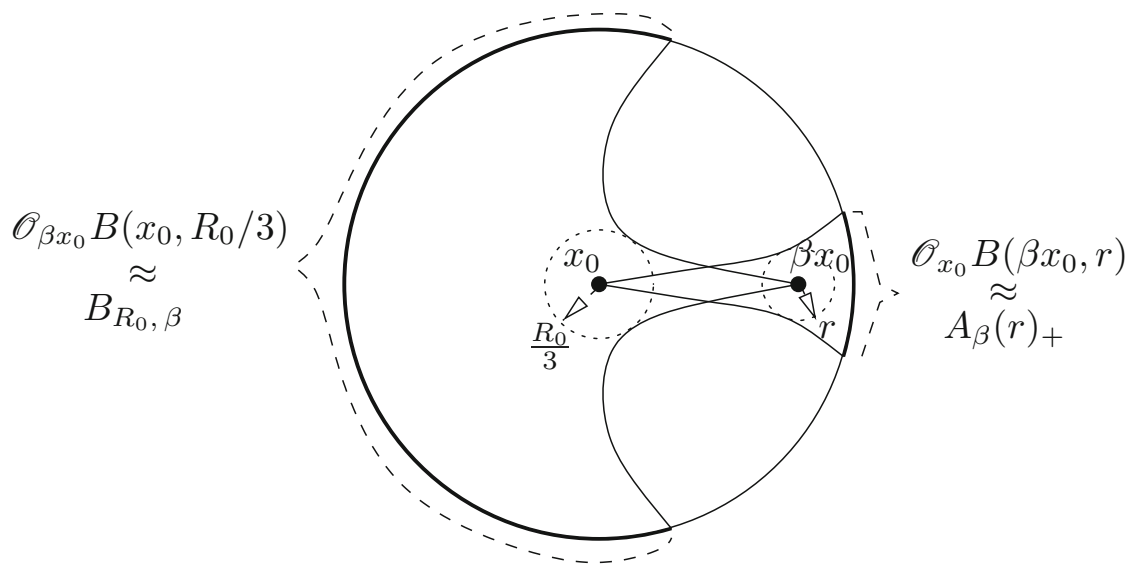

Let us first prove, using Hopf's parametrisation defined by the point $x_{0}$ and the definition of $A_{\beta}(r)$, that for all but finitely many $\beta \in \Gamma$, we have

$$
B_{R_{0}, \beta} \times A_{\beta}(r / 2)_{+} \times\left[-R_{0} / 3, R_{0} / 3\right] \subset A_{\beta}(r) \subset \partial_{\infty} \tilde{M} \times A_{\beta}(r)_{+} \times\left[-R_{0}, R_{0}\right] .
$$

The inclusion on the right hand side is immediate. To prove the other one, let $v \in T^{1} \widetilde{M}$ be such that if $p$ is the closest point to $x_{0}$ on the geodesic line $g_{v}$, then $v_{-} \in B_{R_{0}, \beta}$, $v_{+} \in A_{\beta}(r / 2)_{+}$and $d(\pi(v), p) \leq R_{0} / 3$ (see the picture below). Let us prove that $v \in A_{\beta}(r)$.

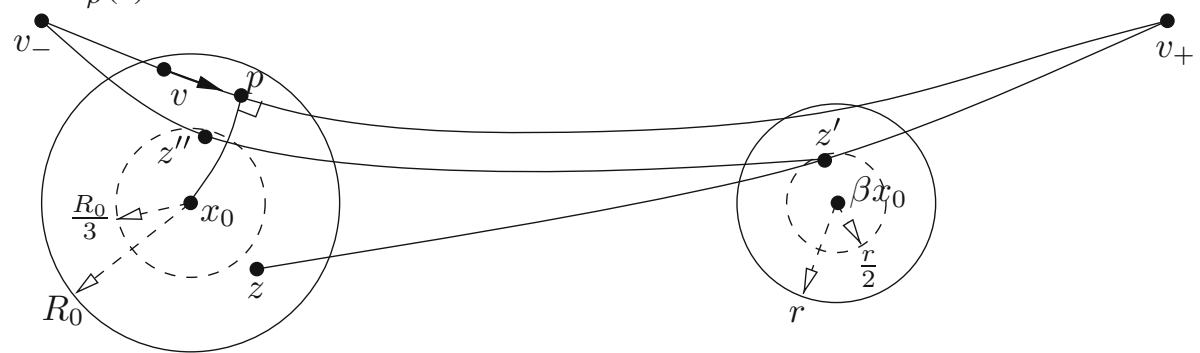

By the definition of $A_{\beta}(r / 2)_{+}$, let $z \in B\left(x_{0}, R_{0}\right)$ be such that the geodesic ray $\left[z, v_{+}[\right.$ meets $B\left(\beta x_{0}, r / 2\right)$ at a point $z^{\prime}$. By the definition of $B_{R_{0}, \beta}$, the geodesic ray $\left[z^{\prime}, v_{-}[\right.$ meets $B\left(x_{0}, R_{0} / 3\right)$ at a point $z^{\prime \prime}$. Since $d\left(z, z^{\prime \prime}\right) \leq 2 R_{0}$, and since by discreteness, for all but finitely many $\beta$ in $\Gamma$, the distance $d\left(z, z^{\prime}\right)$ is big, the angle at $z^{\prime}$ between the geodesic segments $\left[z^{\prime}, z\right]$ and $\left[z^{\prime}, z^{\prime \prime}\right]$ is small, hence the angle at $z^{\prime}$ between the geodesic rays $\left[z^{\prime}, v_{-}\left[\right.\right.$and $\left[z^{\prime}, v_{+}\right.$[ is close to $\pi$. Hence the geodesic line $g_{v}$ between $v_{-}$and $v_{+}$is close to the union of these two rays. In particular, since $g_{v}$ passes close to $z^{\prime} \in B\left(\beta x_{0}, r / 2\right)$, it enters the ball $B\left(\gamma x_{0}, r\right)$, and since it passes close to $z^{\prime \prime} \in B\left(x_{0}, \frac{R_{0}}{3}\right)$, the point $p$ belongs to $B\left(x_{0}, \frac{R_{0}}{2}\right)$ and hence $\pi(v)$ belongs to $B\left(x_{0}, R_{0}\right)$, which proves the result.

Now, for every $v \in A_{\beta}(r)$, since $d\left(\pi(v), x_{0}\right) \leq R_{0}$, the point $x_{0}$ is at distance at most $R_{0}$ from a point on the geodesic line between the endpoints $v_{-}, v_{+}$. Hence by Eq. (6), there exists $c_{6}^{\prime} \geq 0$ (depending only on $R_{0}$, on $\max _{\widetilde{U_{0}}}|\widetilde{F}|<+\infty$, on the Hölder constants of $\widetilde{F}$ and on the bounds of the sectional curvature of $\widetilde{M}$ ) such that, for every $v \in A_{\beta}(r)$, we have

$$
-c_{6}^{\prime} \leq C_{v_{-}}^{F \circ l}\left(x_{0}, \pi(v)\right), C_{v_{+}}^{F}\left(x_{0}, \pi(v)\right) \leq c_{6}^{\prime} .
$$


Therefore, by definition of the Gibbs measure $\widetilde{m}_{F}$, we have, using Eqs. (22) and (24), $e^{-2 c_{6}^{\prime}} \frac{m}{2} \mu_{x_{0}}^{F}\left(A_{\beta}(r / 2)_{+}\right)\left(2 R_{0} / 3\right) \leq \widetilde{m}_{F}\left(A_{\beta}(r)\right) \leq e^{2 c_{6}^{\prime}}\left\|\mu_{x_{0}}^{F \circ \iota}\right\| \mu_{x_{0}}^{F}\left(A_{\beta}(r)_{+}\right)\left(2 R_{0}\right)$.

Hence, by Eq. (23), for some constant $c_{6}^{\prime \prime} \geq 1$, we have

$$
\frac{1}{c_{6}^{\prime \prime}} e^{\int_{x_{0}}^{\beta x_{0}}(\widetilde{F}-\delta)} \leq \widetilde{m}_{F}\left(A_{\beta}(r)\right) \leq c_{6}^{\prime \prime} e^{\int_{x_{0}}^{\beta x_{0}}(\widetilde{F}-\delta)}
$$

For all $[\gamma] \in \Gamma / \Gamma_{0}$ and $\alpha \in \Gamma_{0}$, by Eqs. (15) and (16), if $\beta=\gamma \alpha$, we have

$$
\left|\int_{x_{0}}^{\beta x_{0}}(\widetilde{F}-\delta)-\int_{x_{0}}^{\gamma x_{0}}(\widetilde{F}-\delta)-\int_{x_{0}}^{\alpha x_{0}}(\widetilde{F}-\delta)\right| \leq c_{5}+\delta c_{4}
$$

The result follows.

Note, as it will be important later on, that the contributions of $\gamma$ and of $\alpha$ are decoupled in this Proposition 4.7.

The sets $A_{\gamma \alpha}(r)$ satisfy the following almost disjointness property in shells.

Lemma 4.8. For every $r>0$, there exists $c_{8}=c_{8}(r)>0$ such that for every $k \in \mathbb{N}$, for every subset $P$ of $J_{k}=J_{k}(\psi)$,

$$
\frac{1}{c_{8}} \sum_{([\gamma], \alpha) \in P} \widetilde{m}_{F}\left(A_{\gamma \alpha}(r)\right) \leq \widetilde{m}_{F}\left(\bigcup_{([\gamma], \alpha) \in P} A_{\gamma \alpha}(r)\right) \leq \sum_{([\gamma], \alpha) \in P} \widetilde{m}_{F}\left(A_{\gamma \alpha}(r)\right),
$$

and for every subset $Q$ of $I_{k}$

$$
\frac{1}{c_{8}} \sum_{[\gamma] \in Q} \widetilde{m}_{F}\left(A_{\gamma}(r)\right) \leq \widetilde{m}_{F}\left(\bigcup_{[\gamma] \in Q} A_{\gamma}(r)\right) \leq \sum_{[\gamma] \in Q} \widetilde{m}_{F}\left(A_{\gamma}(r)\right) .
$$

Proof. The inequality on the right hand side of the first claim is immediate. In order to obtain the one on the left hand side, let us prove that there exists $c_{8} \in \mathbb{N}-\{0\}$ such that for all $k \in \mathbb{N}$ and $v \in T^{1} \widetilde{M}$, the number of $([\gamma], \alpha) \in J_{k}$ such that $v \in A_{\gamma \alpha}(r)$ is at most $c_{8}$, which implies the result.

Let $([\gamma], \alpha),\left(\left[\gamma^{\prime}\right], \alpha^{\prime}\right) \in J_{k}$ be such that $v \in A_{\gamma \alpha}(r) \cap A_{\gamma^{\prime} \alpha^{\prime}}(r)$. By Eqs. (14) and (15), and by the definition of $J_{k}$, we have

$$
k+\psi(k)-c_{4} \leq D_{\gamma}+\psi(k)-c_{4} \leq d\left(x_{0}, \gamma x_{0}\right)+d\left(x_{0}, \alpha x_{0}\right)-c_{4} \leq d\left(x_{0}, \gamma \alpha x_{0}\right)
$$

and

$$
\begin{aligned}
d\left(x_{0}, \gamma \alpha x_{0}\right) & \leq d\left(x_{0}, \gamma x_{0}\right)+d\left(x_{0}, \alpha x_{0}\right) \leq D_{\gamma}+\Delta_{0}+\psi(k)+c_{6} \\
& \leq k+\kappa^{\prime}+\Delta_{0}+\psi(k)+c_{6} .
\end{aligned}
$$


Similarly $k+\psi(k)-c_{4} \leq d\left(x_{0}, \gamma^{\prime} \alpha^{\prime} x_{0}\right) \leq k+\psi(k)+\kappa^{\prime}+\Delta_{0}+c_{6}$.

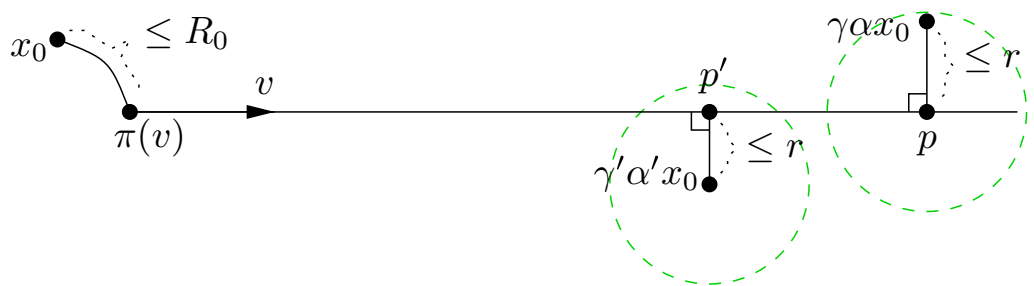

Let $p$ and $p^{\prime}$ be the closest points on $g_{v}\left(\left[0,+\infty[)\right.\right.$ to $\gamma \alpha x_{0}$ and $\gamma^{\prime} \alpha^{\prime} x_{0}$ respectively. They satisfy $d\left(p, \gamma \alpha x_{0}\right), d\left(p^{\prime}, \gamma^{\prime} \alpha^{\prime} x_{0}\right) \leq r$ since $v \in A_{\gamma \alpha}(r) \cap A_{\gamma^{\prime} \alpha^{\prime}}(r)$. We may assume, up to permuting $\gamma \alpha$ and $\gamma^{\prime} \alpha^{\prime}$, that $p^{\prime}$ belongs to the geodesic segment $[\pi(v), p]$. Since closest point maps do not increase the distances, by the triangle inequality, and since $v \in \widetilde{U}_{0}$, we have

$$
\begin{aligned}
d\left(p, p^{\prime}\right) & =d(p, \pi(v))-d\left(\pi(v), p^{\prime}\right) \leq d\left(\gamma \alpha x_{0}, \pi(v)\right)-d\left(\pi(v), p^{\prime}\right) \\
& \leq d\left(\gamma \alpha x_{0}, x_{0}\right)+d\left(x_{0}, \pi(v)\right)-d\left(\gamma^{\prime} \alpha^{\prime} x_{0}, x_{0}\right)+d\left(\pi(v), x_{0}\right)+d\left(p^{\prime}, \gamma^{\prime} \alpha^{\prime} x_{0}\right) \\
& \leq\left(k+\psi(k)+\kappa^{\prime}+\Delta_{0}+c_{6}\right)+R_{0}-\left(k+\psi(k)-c_{4}\right)+R_{0}+r .
\end{aligned}
$$

Hence, again by the triangle inequality,

$$
d\left(\gamma \alpha x_{0}, \gamma^{\prime} \alpha^{\prime} x_{0}\right) \leq \kappa^{\prime}+\Delta_{0}+c_{6}+2 R_{0}+c_{4}+3 r
$$

Now the first claim follows from the discreteness of $\Gamma$, which implies that there are only finitely many elements $\beta$ in $\Gamma$ such that $\beta x_{0}$ belongs to a ball of centre $x_{0}$ with given radius.

The second claim is proven similarly.

The two results above allow to estimate the mass of the $A_{k}(r, \psi)$ 's, as follows.

Proposition 4.9. Assume that there exists $\kappa>0$ such that

$$
\sum_{\gamma \in \Gamma, t \leq d(x, \gamma y)<t+\kappa} e^{\int_{x}^{\gamma y} \widetilde{F}} \asymp e^{\delta t} \text { and } \sum_{\alpha \in \Gamma_{0}, t \leq d(x, \alpha y)<t+\kappa} e^{\int_{x}^{\alpha y} \widetilde{F}} \asymp e^{\delta_{0} t} .
$$

If $r$ is big enough, there exists $c_{9}>0$ such that, for every $k \in \mathbb{N}$, we have

$$
\frac{1}{c_{9}} e^{\psi(k)\left(\delta_{0}-\delta\right)} \leq \widetilde{m}_{F}\left(A_{k}(r, \psi)\right) \leq c_{9} e^{\psi(k)\left(\delta_{0}-\delta\right)}
$$

It follows from this proposition and the assumption on the function $\psi$ that the series $\sum_{k \in \mathbb{N}} \widetilde{m}_{F}\left(A_{k}(r, \psi)\right)$ converges if and only if the integral $\int_{0}^{+\infty} e^{\psi(t)\left(\delta_{0}-\delta\right)} d t$ converges.

Proof. By Eq. (14) and by the first asymptotic assumption in the statement of Proposition 4.9 , there exists $c>0$ such that for every $k \in \mathbb{N}$,

$$
\sum_{[\gamma] \in \Gamma / \Gamma_{0}, k \leq D_{\gamma}<k+\kappa^{\prime}} e^{\int_{x_{0}}^{\gamma x_{0}} \widetilde{F}} \leq \sum_{\gamma \in \Gamma, k \leq d\left(x_{0}, \gamma x_{0}\right)<k+\kappa^{\prime}+\Delta_{0}} e^{\int_{x_{0}}^{\gamma x_{0}} \widetilde{F}} \leq c e^{\delta k} .
$$


By the second asymptotic assumption in Proposition 4.9, since $c_{6} \geq \kappa$, there exists $c^{\prime}>0$ such that, for every $t \in[0,+\infty[$,

$$
\frac{1}{c^{\prime}} e^{\delta_{0} t} \leq \sum_{\alpha \in \Gamma_{0}, t \leq d\left(x_{0}, \gamma x_{0}\right)<t+c_{6}} e^{\int_{x_{0}}^{\alpha x_{0}} \widetilde{F}} \leq c^{\prime} e^{\delta_{0} t} .
$$

Let us first prove the inequality on the right hand side in Proposition 4.9. Let $r$ be big enough and $k \in \mathbb{N}$. Respectively by Lemma 4.8 with $P=J_{k}$ and the definition of $A_{k}(r, \psi)$, by Proposition 4.7, by the definition of $J_{k}$, by Eqs. (26) and (27), we have

$$
\begin{aligned}
m_{F}\left(A_{k}(r, \psi)\right) \leq & \sum_{([\gamma], \alpha) \in J_{k}} \widetilde{m}_{F}\left(A_{\gamma \alpha}(r)\right) \leq c_{7} \sum_{([\gamma], \alpha) \in J_{k}} e^{\int_{x_{0}}^{\gamma x_{0}}(\widetilde{F}-\delta)} e^{\int_{x_{0}}^{\alpha x_{0}}(\widetilde{F}-\delta)} \\
= & c_{7} \sum_{[\gamma] \in \Gamma / \Gamma_{0}, k \leq D_{\gamma}<k+\kappa^{\prime}} e^{\int_{x_{0}}^{\gamma x_{0}}(\widetilde{F}-\delta)} \times \\
& \sum_{\alpha \in \Gamma_{0}, \psi(k) \leq d\left(x_{0}, \alpha x_{0}\right)<\psi(k)+c_{6}} e^{\int_{x_{0}}^{\alpha x_{0}}(\widetilde{F}-\delta)} \\
\leq & c_{7}\left(c e^{\delta k}\right) e^{-\delta k}\left(c^{\prime} e^{\delta_{0} \psi(k)}\right) e^{-\delta \psi(k)}=c_{7} c c^{\prime} e^{\psi(k)\left(\delta_{0}-\delta\right)} .
\end{aligned}
$$

This proves the inequality on the right hand side in Proposition 4.9.

Let us now prove similarly the inequality on the left hand side in Proposition 4.9. By Lemma 4.4, there exists $c^{\prime \prime}>0$ such that, for every $t \in[0,+\infty[$,

$$
\sum_{[\gamma] \in \Gamma / \Gamma_{0}, t \leq D_{\gamma}<t+\kappa^{\prime}} e^{\int_{x_{0}}^{\gamma x_{0}} \widetilde{F}} \geq \frac{1}{c^{\prime \prime}} e^{\delta t} .
$$

Respectively by Lemma 4.8 with $P=J_{k}$ and the definition of $A_{k}(r, \psi)$, by Proposition 4.7 , by the definition of $J_{k}$, by Eqs. (28), (14) and (27), we have

$$
\begin{aligned}
m_{F}\left(A_{k}(r, \psi)\right) \geq & \frac{1}{c_{8}} \sum_{([\gamma], \alpha) \in J_{k}} \tilde{m}_{F}\left(A_{\gamma \alpha}(r)\right) \geq \frac{1}{c_{7} c_{8}} \sum_{([\gamma], \alpha) \in J_{k}} e^{\int_{x_{0}}^{\gamma x_{0}}(\widetilde{F}-\delta)} e^{\int_{x_{0}}^{\alpha x_{0}}(\widetilde{F}-\delta)} \\
= & \frac{1}{c_{7} c_{8}} \sum_{[\gamma] \in \Gamma / \Gamma_{0}, k \leq D_{\gamma}<k+\kappa^{\prime}} e^{\int_{x_{0}}^{\gamma x_{0}}(\widetilde{F}-\delta)} \times \\
& \sum e^{\int_{x_{0}}^{\alpha x_{0}}(\widetilde{F}-\delta)}, \psi \frac{1}{c_{7} c_{8}}\left(\frac{1}{c^{\prime \prime}} e^{\delta k}\right) e^{-\delta\left(k+\kappa^{\prime}+\Delta_{0}\right)}\left(\frac{1}{c^{\prime}} e^{\delta_{0} \psi(k)}\right) e^{-\delta\left(\psi(k)+c_{6}\right)} \\
= & \frac{1}{c_{7} c_{8} c^{\prime} c^{\prime \prime} e^{\delta\left(\kappa^{\prime}+\Delta_{0}+c_{6}\right)}} e^{\psi(k)\left(\delta_{0}-\delta\right)} .
\end{aligned}
$$

This proves Proposition 4.9.

The following result is a quasi-independence property of the sets $A_{k}(r, \psi)$ for $k \in \mathbb{N}$.

Proposition 4.10. Under the hypotheses of Proposition 4.9, there exists a constant $c_{10}>$ 0 such that for every $k \neq k^{\prime}$ in $\mathbb{N}$, if $\psi \geq c_{10}$, we have

$$
\widetilde{m}_{F}\left(A_{k}(r, \psi) \cap A_{k^{\prime}}(r, \psi)\right) \leq c_{10} \widetilde{m}_{F}\left(A_{k}(r, \psi)\right) \widetilde{m}_{F}\left(A_{k^{\prime}}(r, \psi)\right) .
$$


Proof. The proof has two parts, a geometric one and a measure-theoretic one. We state the geometric one as a lemma.

Lemma 4.11. There exist $c_{10}^{\prime}>0$ and $r^{\prime}>r$ such that for every $k<k^{\prime}$ in $\mathbb{N}$, for every $([\gamma], \alpha) \in J_{k}$ and $\left(\left[\gamma^{\prime}\right], \alpha^{\prime}\right) \in J_{k^{\prime}}$, if $\psi \geq c_{10}^{\prime}$ and if $A_{\gamma \alpha}(r)$ meets $A_{\gamma^{\prime} \alpha^{\prime}}(r)$, then $A_{\gamma^{\prime}}(r)$ is contained in $A_{\gamma \alpha}\left(r^{\prime}\right)$.

Proof. Let $k<k^{\prime}$ in $\mathbb{N}$ and $([\gamma], \alpha) \in J_{k}$ and $\left(\left[\gamma^{\prime}\right], \alpha^{\prime}\right) \in J_{k^{\prime}}$. If $A_{\gamma \alpha}(r) \cap A_{\gamma^{\prime} \alpha^{\prime}}(r)$ is non empty, there exists $v \in \widetilde{U_{0}}$ such that $g_{v}(\mathbb{R})$ meets $B\left(\gamma \alpha x_{0}, r\right)$ and $B\left(\gamma^{\prime} \alpha^{\prime} x_{0}, r\right)$. Let $q, q^{\prime}$ be the closest points to $\pi(v)$ on the convex sets $\gamma C_{0}, \gamma^{\prime} C_{0}$. Let $p, p^{\prime}$ be the closest points to $q, q^{\prime}$ on the geodesic ray $g_{v}\left(\left[0,+\infty[)\right.\right.$. Let $x, x^{\prime}$ be the closest points to $\gamma \alpha x_{0}$ and $\gamma^{\prime} \alpha^{\prime} x_{0}$ on $g_{v}([0,+\infty[)$

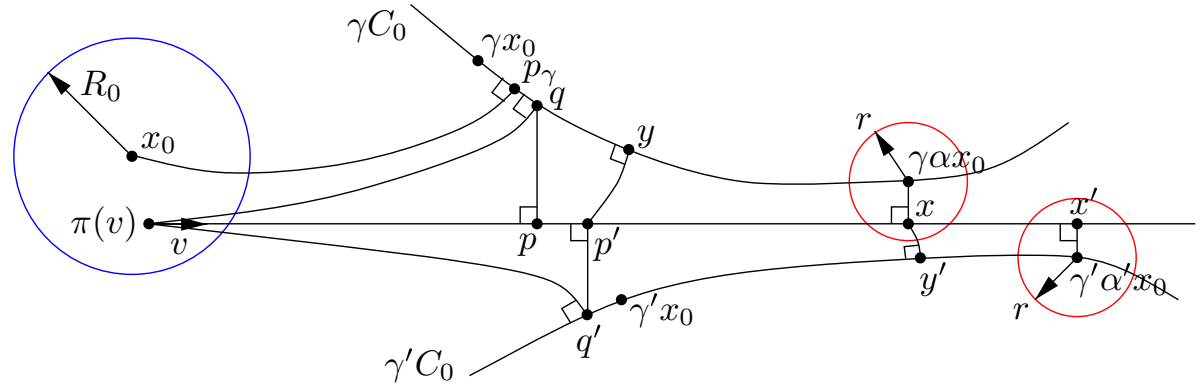

We have $d\left(\gamma \alpha x_{0}, x\right) \leq r$ and $d\left(\gamma^{\prime} \alpha^{\prime} x_{0}, x^{\prime}\right) \leq r$. By the properties of geodesic triangles in CAT $(-1)$-spaces and by the convexity of $C_{0}$, we have $d(p, q), d\left(p^{\prime}, q^{\prime}\right) \leq$ $r+\log (1+\sqrt{2})$. By the choice of the representatives of elements in $\Gamma / \Gamma_{0}$, the closest point $p_{\gamma}$ to $x_{0}$ on $\gamma C_{0}$ is at distance at most $\Delta_{0}$ from $\gamma x_{0}$. Hence, since closest point maps do not increase the distances and by the triangle inequality,

$$
d\left(p, \gamma x_{0}\right) \leq d(p, q)+d\left(q, p_{\gamma}\right)+d\left(p_{\gamma}, \gamma x_{0}\right) \leq r+\log (1+\sqrt{2})+R_{0}+\Delta_{0} .
$$

Hence, by Eq. (14) and the definition of $J_{k}$, with $c=\kappa^{\prime}+2 R_{0}+2 \Delta_{0}+r+\log (1+\sqrt{2})$, we have

$$
\begin{aligned}
& d(\pi(v), p) \leq d\left(\pi(v), x_{0}\right)+d\left(x_{0}, \gamma x_{0}\right)+d\left(\gamma x_{0}, p\right) \\
& \quad \leq R_{0}+\left(D_{\gamma}+\Delta_{0}\right)+d\left(\gamma x_{0}, p\right) \leq k+c,
\end{aligned}
$$

and $d(\pi(v), p) \geq k-c$ by the inverse triangle inequality. Similarly,

$$
k^{\prime}-c \leq d\left(\pi(v), p^{\prime}\right) \leq k^{\prime}+c .
$$

By similar arguments, if $c^{\prime}=R_{0}+\Delta_{0}+2 r+\log (1+\sqrt{2})+c_{6}$, we have

$$
\psi(k)-c^{\prime} \leq d(p, x) \leq \psi(k)+c^{\prime} \text { and } \psi\left(k^{\prime}\right)-c^{\prime} \leq d\left(p^{\prime}, x^{\prime}\right) \leq \psi\left(k^{\prime}\right)+c^{\prime} .
$$

Assume first that $\pi(v), x, p^{\prime}$ are in this order on $g_{v}([0,+\infty[)$. Any geodesic ray, with origin at distance at most $R_{0}$ from $x_{0}$ and passing at distance at most $r$ from $\gamma^{\prime} x_{0}$, passes at distance at most $2 r+\log (1+\sqrt{2})+R_{0}+\Delta_{0}$ from $p^{\prime}$ by the analog for $\gamma^{\prime}$ of Eq. (29), hence by convexity passes at distance at most $c^{\prime \prime}=\max \left\{2 R_{0}, 2 r+\log (1+\sqrt{2})+R_{0}+\Delta_{0}\right\}$ from $x$, thus passes at distance at most $c^{\prime \prime}+r$ from $\gamma \alpha x_{0}$. Therefore, if $r^{\prime} \geq c^{\prime \prime}+r>r$, then $A_{\gamma^{\prime}}(r)$ is contained in $A_{\gamma \alpha}\left(r^{\prime}\right)$. 
Assume now that $\pi(v), p^{\prime}, x$ are in this order on $g_{v}([0,+\infty[)$ (see the picture above). There exists a constant $c_{10}^{\prime}>0$ (depending only the hyperbolicity constant $\log (1+\sqrt{2})$ and on $r$ ) such that if $\psi \geq c_{10}^{\prime}$, then $\pi(v), p, x$ and $\pi(v), p^{\prime}, x^{\prime}$ are in this order on $g_{v}([0,+\infty[)$.

Since $k^{\prime} \geq k$, either $\pi(v), p, p^{\prime}$ are in this order on $g_{v}\left(\left[0,+\infty[)\right.\right.$, or $p^{\prime} \in[\pi(v), p]$ is at distance at most $2 c$ from $p$, since then

$$
d\left(p, p^{\prime}\right)=d(p, x)-d\left(p^{\prime}, x\right) \leq(k+c)-\left(k^{\prime}-c\right) \leq 2 c .
$$

In both cases, by convexity, $p^{\prime}$ is at distance at most $2 c+r+\log (1+\sqrt{2})$ from a point $y$ in $\gamma C_{0}$. Similarly, by Eq. (30) and since $\psi$ satisfies $\psi(s) \leq \psi(t)+c_{3}$ if $s \leq t$, either $\pi(v), x, x^{\prime}$ are in this order on $g_{v}\left(\left[0,+\infty[)\right.\right.$, or $x^{\prime} \in[\pi(v), x]$ is at distance at most $2 c+2 c^{\prime}+c_{3}$ from $x$. In both cases, $x$ is at distance at most $2 c+2 c^{\prime}+c_{3}+r+\log (1+\sqrt{2})$ from a point $y^{\prime}$ in $\gamma^{\prime} C_{0}$.

If for a contradiction $d\left(p^{\prime}, x\right)>R$ for arbitrarily large constants $R$, then the geodesic segments $\left[y, \gamma \alpha x_{0}\right]$ and $\left[q^{\prime}, y^{\prime}\right]$, have endpoints at bounded distance from the long geodesic segment $\left[p^{\prime}, x\right]$. Hence they have their endpoints at bounded distance while being long, if $R$ is large. By hyperbolicity, this implies that $\mathscr{N}_{\epsilon_{0}}\left(\gamma C_{0}\right) \cap \mathscr{N}_{\epsilon_{0}}\left(\gamma^{\prime} C_{0}\right)$ contains a long segment if $R$ is large. Taking $R$ large enough, this contradicts the fact that the diameter of this intersection, since $\gamma^{\prime} \neq \gamma$ in $\Gamma / \Gamma_{0}$, is at most the constant $\kappa_{0}$, as explained in the beginning of the proof of Theorem 4.1.

Therefore $d\left(p^{\prime}, x\right) \leq R$ for some $R \geq 0$. Any geodesic ray, with origin at distance at most $R_{0}$ from $x_{0}$ and passing at distance at most $r$ from $\gamma^{\prime} x_{0}$, passes at distance from $\gamma \alpha x_{0}$ at most

$$
\begin{aligned}
r+d\left(\gamma^{\prime} x_{0}, \gamma \alpha x_{0}\right) & \leq r+d\left(\gamma^{\prime} x_{0}, p^{\prime}\right)+d\left(p^{\prime}, x\right)+d\left(x, \gamma \alpha x_{0}\right) \\
& \leq R+3 r+\log (1+\sqrt{2})+R_{0}+\Delta_{0},
\end{aligned}
$$

by the analog for $\gamma^{\prime}$ of Eq. (29). Therefore, if $r^{\prime} \geq R+3 r+\log (1+\sqrt{2})+R_{0}+\Delta_{0}>r$, then $A_{\gamma^{\prime}}(r)$ is contained in $A_{\gamma \alpha}\left(r^{\prime}\right)$.

Now, let us use Lemma 4.11 to prove Proposition 4.10. Let $k, k^{\prime}$ be elements of $\mathbb{N}$ with $k<k^{\prime}$.

For every $([\gamma], \alpha) \in J_{k}$, let $I_{[\gamma], \alpha, k^{\prime}} \subset I_{k^{\prime}}$ be the set of $\left[\gamma^{\prime}\right] \in \Gamma / \Gamma_{0}$ such that there exists $\alpha^{\prime} \in \Gamma_{0}$ with $\left(\left[\gamma^{\prime}\right], \alpha^{\prime}\right) \in J_{k^{\prime}}$ such that the intersection $A_{\gamma \alpha}(r) \cap A_{\gamma^{\prime} \alpha^{\prime}}(r)$ is non empty. Then respectively by Proposition 4.7 , by the second part of Lemma 4.8 with $Q=I_{[\gamma], \alpha, k^{\prime}}$, by Lemma 4.11 and the definition of $I_{[\gamma], \alpha, k^{\prime}}$, and by Proposition 4.7 (twice), we have

$$
\begin{aligned}
\sum_{\left[\gamma^{\prime}\right] \in I_{[\gamma], \alpha, k^{\prime}}} e^{\int_{x_{0}}^{\gamma^{\prime} x_{0}}(\widetilde{F}-\delta)} & \leq \sum_{\left[\gamma^{\prime}\right] \in I_{[\gamma], \alpha, k^{\prime}}} c_{7}\left(r^{\prime}\right) \widetilde{m}_{F}\left(A_{\gamma^{\prime}}\left(r^{\prime}\right)\right) \\
& \leq c_{7}\left(r^{\prime}\right) c_{8}\left(r^{\prime}\right) \widetilde{m}_{F}\left(\bigcup_{\left[\gamma^{\prime}\right] \in I_{[\gamma], \alpha, k^{\prime}}} A_{\gamma^{\prime}}\left(r^{\prime}\right)\right) \\
& \leq c_{7}\left(r^{\prime}\right) c_{8}\left(r^{\prime}\right) \widetilde{m}_{F}\left(A_{\gamma \alpha}\left(r^{\prime}\right)\right) \\
& \leq c_{7}(r) c_{7}\left(r^{\prime}\right)^{2} c_{8}\left(r^{\prime}\right) \tilde{m}_{F}\left(A_{\gamma \alpha}(r)\right) .
\end{aligned}
$$


By the assumptions of Proposition 4.10, there exists $c>0$ such that for every $t \in \mathbb{R}$,

$$
\sum_{\alpha^{\prime} \in \Gamma_{0}, d\left(x_{0}, \alpha^{\prime} x_{0}\right)<t} e^{\int_{x_{0}}^{\alpha^{\prime} x_{0}} \widetilde{F}} \leq c e^{\delta_{0} t} .
$$

To simplify the notation, let $A_{k}=A_{k}(r, \psi)$. Respectively by the definition of $A_{k}$, by Proposition 4.7 , by Eq. (32), by Eq. (31) with $c^{\prime}=c c_{7}(r)^{2} c_{7}\left(r^{\prime}\right)^{2} c_{8}\left(r^{\prime}\right) e^{c_{6} \delta_{0}}$, and by Proposition 4.9 and Lemma 4.8 with $P=J_{k}$, we have

$$
\begin{aligned}
& \tilde{m}_{F}\left(A_{k} \cap A_{k^{\prime}}\right) \\
& \leq \sum_{([\gamma], \alpha) \in J_{k}} \sum_{\left(\left[\gamma^{\prime}\right], \alpha^{\prime}\right) \in J_{k^{\prime}}, A_{\gamma \alpha}(r) \cap A_{\gamma^{\prime} \alpha^{\prime}}(r) \neq \emptyset} \tilde{m}_{F}\left(A_{\gamma^{\prime} \alpha^{\prime}}(r)\right) \\
& \leq \sum_{([\gamma], \alpha) \in J_{k}} \sum_{\left[\gamma^{\prime}\right] \in I_{[\gamma], \alpha, k^{\prime}}} c_{7}(r) e^{\int_{x_{0}}^{\gamma^{\prime} x_{0}}(\widetilde{F}-\delta)} \sum_{\substack{\alpha^{\prime} \in \Gamma_{0} \\
\psi\left(k^{\prime}\right) \leq d\left(x_{0}, \alpha^{\prime} x_{0}\right)<\psi\left(k^{\prime}\right)+c_{6}}} e^{\int_{x_{0}}^{\alpha^{\prime} x_{0}}(\widetilde{F}-\delta)} \\
& \leq c c_{7}(r) e^{\left(\psi\left(k^{\prime}\right)+c_{6}\right) \delta_{0}-\delta \psi\left(k^{\prime}\right)} \sum_{([\gamma], \alpha) \in J_{k}} \sum_{\left[\gamma^{\prime}\right] \in I_{[\gamma], \alpha, k^{\prime}}} e^{\int_{x_{0}}^{\gamma^{\prime} x_{0}}(\widetilde{F}-\delta)} \\
& \leq c^{\prime} e^{\psi\left(k^{\prime}\right)\left(\delta_{0}-\delta\right)} \sum_{([\gamma], \alpha) \in J_{k}} \tilde{m}_{F}\left(A_{\gamma \alpha}(r)\right) \\
& c_{9} c_{8}(r) \tilde{m}_{F}\left(A_{k^{\prime}}\right) \tilde{m}_{F}\left(A_{k}\right) .
\end{aligned}
$$

This proves Proposition 4.10.

Let us now conclude the proof of Theorem 4.1. The following version of the BorelCantelli Lemma is well-known (see for instance [Spr]).

Proposition 4.12. Let $(Z, v)$ be a measured space with finite nonzero measure. Let $\left(A_{n}\right)_{n \in \mathbb{N}}$ be a sequence of measurable subsets of $Z$ such that there exists $c>0$ with $v\left(A_{n} \cap A_{m}\right) \leq c v\left(A_{n}\right) v\left(A_{m}\right)$ for all distinct $n, m$ in $\mathbb{N}$. Then $v\left(\lim \sup _{n} A_{n}\right)>0$ if and only if the series $\sum_{n \in \mathbb{N}} v\left(A_{n}\right)$ diverges.

We apply this result with $(Z, v)=\left(\widetilde{U_{0}}, \widetilde{m}_{\mid \widetilde{U}_{0}}\right)$, which satisfies the hypothesis if $R_{0}$ is big enough as in the reductions at the beginning of the proof of Theorem 4.1. Let $r=\epsilon_{0}+\Delta_{0}$ and let $c_{5}^{\prime}, c_{5}^{\prime \prime}$ be given by Proposition 4.6.

Assume first that the integral $\int_{0}^{+\infty} e^{\psi(t)\left(\delta_{0}-\delta\right)} d t$ diverges, which is still true if a constant is added to $\psi$. The quasi-independence assumption of Proposition 4.12 is satisfied if $A_{n}=A_{n}\left(r, \psi+c_{10}+c_{5}^{\prime \prime}\right) \subset \widetilde{U_{0}}$, by Proposition 4.10. As claimed after the statement of Proposition 4.9 , the series $\sum_{k \in \mathbb{N}} \widetilde{m}_{F}\left(A_{k}\right)$ diverges. Hence by the above Borel-Cantelli argument, $\lim \sup _{k} A_{k}$ has positive measure. Since $A_{n}\left(r, \psi+c_{10}+c_{5}^{\prime \prime}\right) \subset A_{n}\left(r, \psi+c_{5}^{\prime \prime}\right)$ and by the first claim of Proposition 4.6, the set $\widetilde{E}(\psi)$ has positive $\widetilde{m}_{F}$-measure. Since it is invariant under the geodesic flow and under $\Gamma$, and by ergodicity of the Gibbs measure $m_{F}$, it has full measure.

Conversely, assume that the integral $\int_{0}^{+\infty} e^{\psi(t)\left(\delta_{0}-\delta\right)} d t$ converges, which is still true if a constant is subtracted from $\psi$. Then $\psi(t) \geq c_{5}^{\prime}$ whenever $t$ is large enough. Let $A_{n}=A_{n}\left(r, \psi-c_{5}^{\prime}\right) \subset \widetilde{U_{0}}$. Again by the assertion following the statement of Proposition 4.9 , the series $\sum_{k \in \mathbb{N}} \widetilde{m}_{F}\left(A_{k}\right)$ converges. By the standard Borel-Cantelli 
Lemma, $\lim \sup _{k} A_{k}\left(r, \psi-c_{5}^{\prime}\right)$ has zero $\widetilde{m}_{F}$-measure. By the second claim of Proposition 4.6, the set $\widetilde{E}(\psi) \cap \widetilde{U_{0}}$ has zero $\widetilde{m}_{F}$-measure. Up to taking $R_{0}$ big enough, this implies that $\widetilde{E}(\psi)$ has zero $\widetilde{m}_{F}$-measure.

Remark Let us comment on the range of the numerical constant $\delta-\delta_{0}$, crucial for the dichotomy in Theorem 4.1, as the potential $F$ varies. We only consider the case when $C_{0}$ is the translation axis of a loxodromic element of $\Gamma$, so that by Remark (2) following the statement of Theorem 4.1, we have, with $\overline{C_{0}}$ the image of $C_{0}$ in $M=\Gamma \backslash \widetilde{\Gamma}$,

$$
\delta-\delta_{0}=P(F)-P\left(F_{\mid T^{1} \overline{C_{0}}}\right) .
$$

Proposition 4.13.(1) The map $F \mapsto P(F)-P\left(F_{\mid T^{1}} \bar{C}_{0}\right)$ is 1-Lipschitz for the uniform norm on bounded potentials.

(2) The set of real numbers $P(F)-P\left(F_{\mid T^{1}} \bar{C}_{0}\right)$, as $\widetilde{F}$ varies in the set of $\Gamma$-invariant bounded Hölder functions on $T^{1} \widetilde{M}$, is equal to $] 0,+\infty[$.

Proof. For the first observation, the 1-Lipschitz dependence of $P\left(F_{\mid T^{1}} \overline{C_{0}}\right)$ on $F$ is immediate by Eq. (2), and so it suffices to prove it for $P(F)$. This is a direct consequence of our definition of the pressure in Eq. (1). More precisely, given $F_{1}, F_{2}$ two bounded $\Gamma$-invariant Hölder-continuous functions on $T^{1} \vec{M}$, for every $\epsilon>0$, we can choose $m_{1}, m_{2} \in \mathscr{M}$ satisfying

$$
h\left(m_{1}\right)+\int F_{1} d m_{1} \geq P\left(F_{1}\right)-\epsilon \quad \text { and } \quad h\left(m_{2}\right)+\int F_{2} d m_{2} \geq P\left(F_{2}\right)-\epsilon .
$$

Using the definition of pressure again, we have that

$$
P\left(F_{1}\right) \geq h\left(m_{2}\right)+\int F_{1} d m_{2} \text { and } P\left(F_{2}\right) \geq h\left(m_{1}\right)+\int F_{2} d m_{1} .
$$

Comparing these four inequalities gives that

$$
\int\left(F_{1}-F_{2}\right) d m_{1} \geq P\left(F_{1}\right)-P\left(F_{2}\right)-\epsilon \text { and } \int\left(F_{2}-F_{1}\right) d m_{2} \geq P\left(F_{2}\right)-P\left(F_{1}\right)-\epsilon,
$$

from which we deduce $\left|P\left(F_{1}\right)-P\left(F_{2}\right)\right| \leq\left\|F_{1}-F_{2}\right\|_{\infty}+\epsilon$. Letting $\epsilon \rightarrow 0$, this proves that $F \mapsto P(F)$ is 1-Lipschitz.

For the second observation, first note that $P(F)-P\left(F_{\mid T^{1}} \overline{C_{0}}\right)=\delta-\delta_{0}$ is positive by Lemma 4.2. It now suffices to find two potentials $F, F^{\prime}$ for which $P(F)-P\left(F_{\mid T^{1} \overline{C_{0}}}\right)$ can be arbitrarily large and $P\left(F^{\prime}\right)-P\left(F^{\prime}{ }_{\mid T^{1}} \bar{C}_{0}\right)$ can be arbitrarily close to 0 .

Given any $L>0$ and a second distinct closed geodesic $\overline{C_{1}}$ (which exists since $\Gamma$ is nonelementary), we can choose a bounded potential $F$ on $T^{1} M$ which is constant with values $L$ and 0 on $T^{1} \overline{C_{1}}$ and $T^{1} \overline{C_{0}}$, respectively. If $m_{C_{1}}$ denotes a probability measure supported on $T^{1} \overline{C_{1}}$ and invariant under the geodesic flow, then by the definition of the pressure, we have that $P\left(F_{\mid T^{1} \overline{C_{0}}}\right)=0$ and $P(F) \geq h_{m_{C_{1}}}\left(g^{1}\right)+\int F d m_{C_{1}}=L$, as required.

Finally, given any $\eta>0$, we want to construct a bounded potential $F^{\prime}$ on $T^{1} M$ satisfying $P\left(F^{\prime}\right)-P\left(F^{\prime}{ }_{\mid T^{1}} \overline{C_{0}}\right)<\eta$. For every $\epsilon>0$, let $A_{0}=\left\{v \in T^{1} M: d\left(v, T^{1} \overline{C_{0}}\right)<\epsilon\right\}$. We choose $\epsilon \in] 0, \frac{1}{e}$ [ small enough, so that $-(1-\epsilon) \log (1-\epsilon)-2 \epsilon \log \epsilon+4 \epsilon \log 2<\eta$. We choose $K>h_{\text {top }}\left(g^{1}\right) / \epsilon^{2}$, and we define a bounded potential $F^{\prime}$ on $T^{1} M$ by 
$F^{\prime}(v)=-K \min \left\{d\left(v, T^{1} \overline{C_{0}}\right), 1\right\} \leq 0$. Given any $m \in \mathscr{M}$, we can consider two cases: Either (a) $m\left(T^{1} M-A_{0}\right)>\epsilon$ or (b) $m\left(A_{0}\right) \geq 1-\epsilon$. In case (a), we have that

$$
\begin{aligned}
h(m)+\int F^{\prime} d m & \leq h_{\mathrm{top}}\left(g^{1}\right)+m\left(T^{1} M-A_{0}\right) \max _{v \in T^{1} M-A_{0}} F^{\prime}(v) \\
& \leq h_{\mathrm{top}}\left(g^{1}\right)-K \epsilon^{2}<0 .
\end{aligned}
$$

In case (b), we can choose a measurable partition $\alpha=\left\{A_{n}\right\}_{n \in \mathbb{N}}$ of $T^{1} M$, such that:

- $\alpha$ is generating, that is, the Borel $\sigma$-algebra is the smallest $\sigma$-algebra containing $g^{t_{1}} A_{i_{1}} \cap \cdots \cap g^{t_{k}} A_{i_{k}}$, for all $k, i_{1}, \cdots, i_{k} \in \mathbb{N}$ and $t_{1}, \cdots, t_{k} \in \mathbb{R}$;

- for $n \geq 1$, we have $m\left(A_{n}\right) \leq \epsilon / 2^{n-1}$ (note that $m\left(\bigcup_{n=1}^{+\infty} A_{n}\right)=1-m\left(A_{0}\right) \leq \epsilon$ ).

If $M$ were compact, then a sufficient condition for the partition to be generating would be that each element $A_{n}$, for $n \geq 1$, has diameter smaller than the injectivity radius of $M$. (At the level of the geodesic flow, this is related to choosing the diameter smaller than the expansivity constant). More generally, we can assume that each $A_{n}$ is the union of suitably separated components, each of which has diameter smaller than the injectivity radius of points in that component. In particular, with $H_{m}(\alpha)$ the entropy of the partition $\alpha$ with respect to $m$, we can then bound

$$
\begin{aligned}
h(m)+\int F^{\prime} d \mu & \leq h(m) \leq H_{m}(\alpha) \\
& \leq-m\left(A_{0}\right) \log m\left(A_{0}\right)-\sum_{n=1}^{+\infty} m\left(A_{n}\right) \log m\left(A_{n}\right) \\
& \leq-(1-\epsilon) \log (1-\epsilon)-\sum_{n=1}^{+\infty} \frac{\epsilon}{2^{n-1}} \log \frac{\epsilon}{2^{n-1}} \\
& =-(1-\epsilon) \log (1-\epsilon)-2 \epsilon \log \epsilon+4 \epsilon \log 2<\eta .
\end{aligned}
$$

In either case, we have that $h(m)+\int F^{\prime} d m<\eta$ and from the definition, $P\left(F^{\prime}\right)-$ $P\left(F^{\prime}{ }_{\mid T^{1}} \overline{C_{0}}\right)=P\left(F^{\prime}\right)<\eta$, as required.

Let us now give the main corollary of Theorem 4.1, our logarithm law for Gibbs measures.

Define the penetration map $\widetilde{\mathfrak{p}}: T^{1} \widetilde{M} \times \mathbb{R} \rightarrow[0,+\infty]$ of the geodesic lines inside $\Gamma \mathscr{N}_{\epsilon_{0}} C_{0}$ by $\widetilde{\mathfrak{p}}(v, t)=0$ if $\pi\left(\phi_{t} v\right) \notin \Gamma \mathscr{N}_{\epsilon_{0}} C_{0}$, and otherwise $\widetilde{\mathfrak{p}}(v, t)$ is the maximal length of an interval $I$ in $\mathbb{R}$ containing $t$ such that there exists $\gamma \in \Gamma$ with $\pi\left(\phi_{s} v\right) \in \gamma \mathscr{N}_{\epsilon_{0}} C_{0}$ for every $s \in I$. The next result implies Corollary 1.3 using Remark (2) following Theorem 4.1.

Corollary 4.14. Under the assumptions of Theorem 4.1, for $\widetilde{m}_{F}$-almost every $v \in T^{1} \tilde{M}$, we have

$$
\limsup _{t \rightarrow+\infty} \frac{\widetilde{\mathfrak{p}}(v, t)}{\log t}=\frac{1}{\delta-\delta_{0}} .
$$

Proof. The proof is a standard deduction from Theorem 4.1 using the Lipschitz functions $\psi_{n}: t \mapsto \kappa \log (1+t)$ for $\kappa=\frac{1}{\delta-\delta_{0}} \pm \frac{1}{n}$, see for instance the proof of [HP2, Theo. 5.6]. 
We end this section by giving a corollary of Theorem 4.1 in the special case when $\widetilde{M}$ has constant sectional curvature, in a form which is suitable for the arithmetic applications in the next section. We will use the upper halfspace model of the real hyperbolic $n$-space $\mathbb{H}_{\mathbb{R}}^{n}$, whose boundary at infinity is $\partial_{\infty} \mathbb{H}_{\mathbb{R}}^{n}=\mathbb{R}^{n-1} \cup\{\infty\}$, and we endow $\mathbb{R}^{n-1}$ with the usual Euclidean norm $\|\cdot\|$ and its associated distance. We denote by $x_{0}$ the point $\left.(0,1) \in \mathbb{R}^{n-1} \times\right] 0,+\infty$ [. If $\alpha$ is a fixed point of a hyperbolic element $\gamma$ of a given discrete group of isometries of $\mathbb{H}_{\mathbb{R}}^{n}$, we denote by $\alpha^{\sigma}$ its other fixed point, which does not depend on $\gamma$.

Corollary 4.15. Let $\Gamma$ be a nonelementary discrete group of isometries of $\mathbb{H}_{\mathbb{R}}^{n}$, with non arithmetic length spectrum. Let $\widetilde{F}: T^{1} \mathbb{H}_{\mathbb{R}}^{n} \rightarrow \mathbb{R}$ be a $\Gamma$-invariant Hölder-continuous map, with $\delta=\delta_{\Gamma, F}$ and $m_{F}$ finite. Let $\gamma_{0}$ be a hyperbolic element of $\Gamma$, let $\Gamma_{0} \underset{\sim}{\text { be the }}$ stabiliser in $\Gamma$ of its translation axis, let $F_{0}: \Gamma_{0} \backslash \widetilde{M} \rightarrow \mathbb{R}$ be the map induced by $\widetilde{F}$, and let $\delta_{0}=\delta_{\Gamma_{0}}, F_{0}$. Let $\mathscr{R}_{\gamma_{0}}$ be the set of fixed points in $\mathbb{R}^{n-1} \cup\{\infty\}$ of the conjugates in $\Gamma$ of $\gamma_{0}$. Let $\left.\left.\left.\left.\phi:\right] 0,1\right] \rightarrow\right] 0,1\right]$ be a measurable map, such that there exist $\left.c_{2}^{\prime}, c_{3}^{\prime} \in\right] 0,1[$ such that for every $\left.s, t \in] 0, c_{2}^{\prime}\right]$, if $s \geq c_{2}^{\prime} t$, then $\phi(s) \geq c_{3}^{\prime} \phi(t)$. If $\int_{0}^{1} \phi^{\delta-\delta_{0}}(s) / s d s$ diverges (respectively converges), then $\mu_{x_{0}}^{F}$-almost every (respectively no) point in $\mathbb{R}^{n-1}$ belongs to infinitely many Euclidean balls of centre $\alpha$ and radius $\left\|\alpha-\alpha^{\sigma}\right\| \phi\left(\left\|\alpha-\alpha^{\sigma}\right\|\right)$, as $\alpha$ ranges over $\mathscr{R}_{\gamma_{0}}$.

Proof. Recall that the hyperbolic distance between the horizontal horosphere at Euclidean height 1 in $\mathbb{H}_{\mathbb{R}}^{n}$ and a disjoint geodesic line with endpoints $x$ and $y$ is $-\log \frac{\|x-y\|}{2}$, by a standard hyperbolic distance computation. By the triangle inequality and the discreteness of $\Gamma$, for every compact subset $K$ of $\mathbb{R}^{n-1}$, there exists $c>0$ such that for every $\alpha \in \mathscr{R}_{\gamma_{0}} \cap K$ except finitely many of them, we have $\left\|\alpha-\alpha^{\sigma}\right\| \leq 1$ and, with $C_{\alpha}$ the geodesic line with endpoints $\alpha, \alpha^{\sigma}$,

$$
\left|d\left(x_{0}, C_{\alpha}\right)-\right| \log \frac{\left\|\alpha-\alpha^{\sigma}\right\|}{2}|| \leq c .
$$

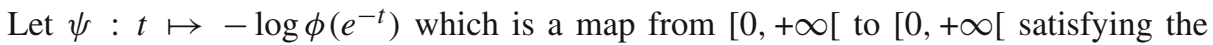
assumption of the beginning of Sect. 4 (with $c_{2}=-\log c_{2}^{\prime}>0$ and $c_{3}=-\log c_{3}^{\prime}>0$ ).

As in [HP2, Lem. 5.2] (and since the Hamenstädt distance on $\partial_{\infty} \mathbb{H}_{\mathbb{R}}^{n}-\{\infty\}=\mathbb{R}^{n-1}$ is a multiple of the Euclidean distance), there exists a constant $c^{\prime} \geq 1$ such that for every $v \in T^{1} \widetilde{M}$ such that $v_{+} \in K-\left(\mathscr{R}_{\gamma_{0}} \cap K\right)$, we have

- if $v$ is $\left(\epsilon_{0}, \psi\right)$-Liouville for $\left(\Gamma, \Gamma_{0}\right)$, then $v_{+}$belongs to infinitely many balls of centre $\alpha$ and radius $c^{\prime} e^{-d\left(x_{0}, C_{\alpha}\right)-\psi\left(d\left(x_{0}, C_{\alpha}\right)\right)}$, as $\alpha$ ranges over $\mathscr{R}_{\gamma_{0}}$.

- if $v_{+}$belongs to infinitely many balls of centre $\alpha$ and radius $\frac{1}{c^{\prime}} e^{-d\left(x_{0}, C_{\alpha}\right)-\psi\left(d\left(x_{0}, C_{\alpha}\right)\right)}$, as $\alpha$ ranges over $\mathscr{R}_{\gamma_{0}}$, then $v$ is $\left(\epsilon_{0}, \psi\right)$-Liouville for $\left(\Gamma, \Gamma_{0}\right)$.

By Eq. (33), there exists $c^{\prime \prime} \geq 1$ such that, for every $\alpha \in \mathscr{R}_{\gamma_{0}} \cap K$,

$$
\frac{1}{c^{\prime \prime}}\left\|\alpha-\alpha^{\sigma}\right\| \phi\left(\left\|\alpha-\alpha^{\sigma}\right\|\right) \leq e^{-d\left(x_{0}, C_{\alpha}\right)-\psi\left(d\left(x_{0}, C_{\alpha}\right)\right)} \leq c^{\prime \prime}\left\|\alpha-\alpha^{\sigma}\right\| \phi\left(\left\|\alpha-\alpha^{\sigma}\right\|\right) .
$$

Since $\int_{0}^{+\infty} e^{\psi(t)\left(\delta_{0}-\delta\right)} d t=\int_{0}^{1} \phi^{\delta-\delta_{0}}(s) / s d s$, the result follows from Theorem 4.1, whose hypotheses on sum asymptotics are satisfied by the first remark following its statement (since the curvature of $\widetilde{M}$ is constant).

Remark As in [PaP2], replacing $\mathbb{H}_{\mathbb{R}}^{n}$ by the Siegel domain model of the complex hyperbolic space $\mathbb{H}_{\mathbb{C}}^{n}$, replacing $\mathbb{R}^{n-1}$ endowed with the Euclidean distance $\|x-y\|$ by the Heisenberg group endowed with the Cygan distance $d_{\text {Cyg }}(x, y)$, the same result holds. 


\section{Arithmetic Applications}

Let $K$ be either the field $\mathbb{Q}$ or an imaginary quadratic extension of $\mathbb{Q}$, and correspondingly, let $\widehat{K}$ be either $\mathbb{R}$ or $\mathbb{C}$. Let $\mathscr{O}_{K}$ be the ring of integers of $K$. By quadratic irrational, we mean an element in $\widehat{K}$ which is quadratic irrational over $K$. For every quadratic irrational $\alpha \in \widehat{K}$, let $\alpha^{\sigma}$ be its Galois conjugate over $K$.

The group $\operatorname{PSL}_{2}(\widehat{K})$ acts on $\mathbb{P}^{1}(K)=\widehat{K} \cup\{\infty\}$ by homographies, and its subgroup $\mathrm{PSL}_{2}\left(\mathscr{O}_{K}\right)$ preserves the set $K$ and the set of quadratic irrationals. Though it acts transitively on the former set, it does not act transitively on the latter one. Note that, for every quadratic irrational $\alpha$ and every $\gamma \in \operatorname{PSL}_{2}\left(\mathscr{O}_{K}\right)$, we have $(\gamma \cdot \alpha)^{\sigma}=\gamma \cdot\left(\alpha^{\sigma}\right)$.

Let us fix a finite index subgroup $\Gamma$ of $\operatorname{PSL}_{2}\left(\mathscr{O}_{K}\right)$, for instance a congruence subgroup. We are interested in the approximation of elements of $\widehat{K}$ by elements in the orbit under $\Gamma$ of a fixed quadratic irrational and of its Galois conjugate.

For every quadratic irrational $\alpha \in \widehat{K}$, let $\mathscr{E}_{\alpha}, \Gamma$ be the (countable, dense in $\widehat{K}$ ) set $\Gamma \cdot\left\{\alpha, \alpha^{\sigma}\right\}$, endowed with its Fréchet filter, and let

$$
h(\alpha)=\frac{2}{\left|\alpha-\alpha^{\sigma}\right|} .
$$

We refer to $[\mathrm{PaP} 2, \S 6.1]$ and $[\mathrm{PaP} 3, \S 4.1]$ for motivations on this complexity $h(\alpha)$ of a quadratic irrational $\alpha$, as well as for other algebraic expressions and comparisons to other algebraic heights. For instance, if $K=\mathbb{Q}, \Gamma=\mathrm{PSL}_{2}(\mathbb{Z})$ and $\alpha$ is the Golden Ratio $\frac{1+\sqrt{5}}{2}$, then $\mathscr{E}_{\alpha, \Gamma}$ is the set of real numbers whose continued fraction expansion ends with an infinite string of 1 's.

Recall that a map $f:[0,+\infty[\rightarrow] 0,+\infty$ [ is slowly varying if it is measurable and if there exist constants $B>0$ and $A \geq 1$ such that for every $x, y$ in $\mathbb{R}_{+}$, if $|x-y| \leq B$, then $f(y) \leq A f(x)$. Recall that this implies that $f$ is locally bounded, hence it is locally integrable; also, if $\log f$ is Lipschitz, then $f$ is slowly varying.

Theorem 5.1. Let $\alpha_{0} \in \widehat{K}$ be a fixed quadratic irrational and let $\gamma_{0} \in \Gamma$ be a primitive element of $\Gamma$ fixing $\alpha_{0}$ with $\left|\gamma_{0}^{\prime}\left(\alpha_{0}\right)\right|>1$. Let $\mu_{x_{0}}^{F}$ be a Patterson measure on $\widehat{K} \cup\{\infty\}$ associated with a potential $\widetilde{F}$ for $\Gamma$ such that $\delta=\delta_{\Gamma, F}$ and $m_{F}$ are finite. Let $\delta_{0}$ be the critical exponent of $\gamma_{0}^{\mathbb{Z}}$ for $\widetilde{F}$. Let $\varphi:\left[0,+\infty[\rightarrow] 0,+\infty\left[\right.\right.$ be a map such that $t \mapsto \varphi\left(e^{t}\right)$ is slowly varying. If the integral $\int_{1}^{+\infty} \varphi(t)^{\delta-\delta_{0}} / t d t$ diverges (resp. converges), then for $\mu_{x_{0}}^{F}$-almost every $x \in \widehat{K}$,

$$
\liminf _{r \in \mathscr{E}_{\alpha_{0}, \Gamma}} \frac{h(r)}{\varphi(h(r))}|x-r|=0(\text { resp. }=+\infty) .
$$

When $F=0$, this result is due to [PaP2, Theo. 6.4 (4)].

Proof. Let us first give some details on the notation of this theorem. Recall (see for instance [PaP2, Lem. 6.2]), that the quadratic irrationals in $\widehat{K}$ are exactly the fixed points of the loxodromic elements of $\operatorname{PSL}_{2}\left(\mathscr{O}_{K}\right)$, hence of $\Gamma$, since $\Gamma$ has finite index in $\operatorname{PSL}_{2}\left(\mathscr{O}_{K}\right)$. Hence an element $\gamma_{0}$ as in the statement exists, it is the unique (up to multiplication by an element of $\Gamma_{0}$ pointwise fixing the translation axis of $\gamma_{0}$ ) primitive loxodromic element of $\Gamma$ with attractive fixed point $\alpha_{0}$.

Let $\widetilde{M}$ be the real hyperbolic plane $\mathbb{H}_{\mathbb{R}}^{2}$ if $\widehat{K}=\mathbb{R}$ and the real hyperbolic space $\mathbb{H}_{\mathbb{R}}^{3}$ if $\widehat{K}=\mathbb{C}$. We fix a point $x_{0}$ in $\widetilde{M}$. Note that $\partial_{\infty} \widetilde{M} \equiv \widehat{K} \cup\{\infty\}$, and $\Gamma$ is a discrete group of isometries (actually an arithmetic lattice) of $\widetilde{M}$, so that a $\Gamma$-invariant potential 
$\widetilde{F}$ on $T^{1} \widetilde{M}$ with $\delta=\delta_{\Gamma, F}$ does define a Patterson measure $\mu_{x_{0}}^{F}$ seen from $x_{0}$ (unique up to scalar multiple if $m_{F}$ is finite) on $\widehat{K} \cup\{\infty\}$, see Sect. 2. Let $\Gamma_{0}$ be the stabiliser of $\left\{\alpha_{0}, \alpha_{0}{ }^{\sigma}\right\}$ in $\Gamma$ (that is of the translation axis of $\gamma_{0}$ ), and let $F_{0}: \Gamma_{0} \backslash T^{1} \widetilde{M} \rightarrow \mathbb{R}$ be the map induced by $\widetilde{F}$. Since $\gamma_{0}^{\mathbb{Z}}$ has finite index in $\Gamma_{0}$, the critical exponent $\delta_{0}$ is equal to $\delta_{\Gamma_{0}}, F_{0}$. Note that $\mathscr{E}_{\alpha_{0}, \Gamma}$ is exactly the set of fixed points of the conjugates of $\gamma_{0}$ in $\Gamma$.

We may assume that $\varphi \leq 1$. Define $\phi: s \mapsto \varphi\left(\frac{2}{s}\right)$, which is a measurable map from ] $0,1]$ to $] 0,1[$. The result then follows from Corollary 4.15.

To conclude, let us give a proof of the last statement of the Introduction.

Proof of Corollary 1.4. It is well known that $\Gamma_{a, b}$ is a uniform lattice in $\mathrm{SL}_{2}(\mathbb{R})$ (see for instance [Kat, §5.2] or [BeP, §8.5]): it is a Fuschian group derived from the quaternion algebra $\left(\frac{a, b}{\mathbb{Q}}\right)$ over $\mathbb{Q}$, which is a division algebra by the nonexistence of nonzero integer solutions to $x^{2}-a y^{2}-b z^{2}=0$, hence to $x^{2}-a y^{2}-b z^{2}+a b t^{2}=0$ by [BeP, Lem. 8.17]. Let $\Gamma=\overline{\Gamma_{a, b}}$ be the image of $\Gamma_{a, b}$ in $\operatorname{PSL}_{2}(\mathbb{R})$, which is a cocompact group of isometries of $\widetilde{M}=\mathbb{H}_{\mathbb{R}}^{2}$, whose action on $\partial_{\infty} \mathbb{H}_{\mathbb{R}}^{2}=\mathbb{P}_{1}(\mathbb{R})$ is the action by homographies. If $\gamma_{0}=\left(\begin{array}{cc}x+y \sqrt{a} & z-t \sqrt{a} \\ b(z+t \sqrt{a}) & x-y \sqrt{a}\end{array}\right)$ with $(x, y, z, t) \in \mathbb{Z}^{4}$, then tr $\gamma_{0}=2 x$. Hence $\left|\operatorname{tr} \gamma_{0}\right|>2$ by the assumptions, that is, the image of $\gamma_{0}$ in $\Gamma$, that we again denote by $\gamma_{0}$, is hyperbolic. It is well known that its translation length $\ell\left(\gamma_{0}\right)$ satisfies (see for instance [Bea, page 173])

$$
\cosh \frac{\ell\left(\gamma_{0}\right)}{2}=\frac{\left|\operatorname{tr} \gamma_{0}\right|}{2}
$$

Let us fix $x_{0} \in \mathbb{H}_{\mathbb{R}}^{2}$. By Proposition 3.6, let $\widetilde{F}: T^{1} \widetilde{M} \rightarrow \mathbb{R}$ be a $\Gamma$-invariant Höldercontinuous map such that $\mu$ and $\mu_{x_{0}}^{F}$ have the same measure class. Since the conclusion of Corollary 1.4 depends only on the measure class of $\mu$, and since $\frac{d\left(\gamma^{-1}\right)_{*} \mu_{x_{0}}^{F}}{d \mu_{x_{0}}^{F}}\left(\gamma^{+}\right)=$ $\frac{d\left(\gamma^{-1}\right)_{*} \mu}{d \mu}\left(\gamma^{+}\right)$for every hyperbolic element $\gamma \in \Gamma$ by [Led3, Théo. 1.c], as seen in the proof of Proposition 3.6, we may assume that $\mu=\mu_{x_{0}}^{F}$. Since $\Gamma$ is cocompact, both $\delta=\delta_{\Gamma, F}$ and $m_{F}$ are finite. Let $F_{0}: \Gamma_{0} \backslash T^{1} \widetilde{M} \rightarrow \mathbb{R}$ be the map induced by $\widetilde{F}$, and let $\delta_{0}=\delta_{\Gamma_{0}}, F_{0}$. By Remark (2) following the statement of Theorem 4.1 and by Eq. (12), we have

$$
\begin{aligned}
\delta_{0} & =\frac{\max \left\{\operatorname{Per}_{F}(\gamma), \operatorname{Per}_{F}\left(\gamma^{-1}\right)\right\}}{\ell\left(\gamma_{0}\right)} \\
& =\frac{1}{2 \operatorname{arcosh}\left(\frac{\left|\operatorname{tr} \gamma_{0}\right|}{2}\right)} \max \left\{\frac{d\left(\gamma_{0}^{-1}\right)_{*} \mu}{d \mu}\left(\gamma_{0}^{+}\right), \frac{d\left(\gamma_{0}\right)_{*} \mu}{d \mu}\left(\gamma_{0}^{-}\right)\right\}
\end{aligned}
$$


Since $\Gamma$ is cocompact, by Bowen's period counting theorem (see for instance [PPS, Theo. 4.7]), again by Eq. (12), and by the change of variable $s=2 \cosh \frac{t}{2}$, we have

$$
\begin{aligned}
\delta & =\delta_{\Gamma, F}=\lim _{t \rightarrow+\infty} \frac{1}{t} \log \sum_{\gamma \in \Gamma, 0<\ell(\gamma) \leq t} e^{\operatorname{Per}_{F}(\gamma)} \\
& =\lim _{t \rightarrow+\infty} \frac{1}{t} \log \sum_{\gamma \in \Gamma_{a, b}, \operatorname{tr}(\gamma) \neq 0, \pm 2,2 \operatorname{arcosh}\left(\frac{|\operatorname{tr} \gamma|}{2}\right) \leq t} \frac{d\left(\gamma^{-1}\right)_{*} \mu}{d \mu}\left(\gamma^{+}\right) \\
& =\lim _{s \rightarrow+\infty} \frac{1}{2 \log s} \log \sum_{\gamma \in \Gamma_{a, b}, 2<|\operatorname{tr}(\gamma)| \leq s} \frac{d\left(\gamma^{-1}\right)_{*} \mu}{d \mu}\left(\gamma^{+}\right) .
\end{aligned}
$$

For all $s \geq 0$ and $\epsilon>0$, let $\phi:] 0,1] \rightarrow] 0,1]$ be the map $t \mapsto \min \left\{1, \epsilon(-\log t)^{-s}\right\}$, so that $\int_{0}^{1} \phi^{\delta-\delta_{0}}(t) / t d t$ diverges if and only if $s \leq \frac{1}{\delta-\delta_{0}}$.

By Corollary 4.15, we hence have that if $s \leq \frac{1}{\delta-\delta_{0}}$ (resp. $s>\frac{1}{\delta-\delta_{0}}$ ), then, for $\mu$-almost every $x \in \mathbb{R}$,

$$
\liminf _{\alpha \in \Gamma_{a, b} \cdot\left\{\gamma_{0}^{-}, \gamma_{0}^{+}\right\},\left|\alpha-\alpha^{\sigma}\right| \rightarrow 0} \frac{|x-\alpha|}{\left|\alpha-\alpha^{\sigma}\right|\left(-\log \left|\alpha-\alpha^{\sigma}\right|\right)^{-s}} \leq \frac{1}{\epsilon} \quad\left(\text { resp. } \geq \frac{1}{\epsilon}\right) .
$$

By taking $\epsilon=k$ (resp. $\epsilon=\frac{1}{k}$ ) for $k \in \mathbb{N}$ tending to $+\infty$, this proves the result.

Acknowledgments. The second author thanks the École Normale Supérieure for an Invited Professor position in 2009 where this work was started, and the Université Paris-Sud (Orsay) for an Invited Professor position in 2014 where this work was completed.

Open Access This article is distributed under the terms of the Creative Commons Attribution 4.0 International License (http://creativecommons.org/licenses/by/4.0/), which permits unrestricted use, distribution, and reproduction in any medium, provided you give appropriate credit to the original author(s) and the source, provide a link to the Creative Commons license, and indicate if changes were made.

\section{References}

[Bab] Babillot, M.: On the mixing property for hyperbolic systems. Israel J. Math. 129, 61-76 (2002)

[Bea] Beardon, A.F.: The Geometry of Discrete Groups. Graduate Texts in Mathematics, vol 91. Springer, UK (1983)

[BeP] Benoist, Y., Paulin, F.: Systèmes dynamiques élémentaires. Notes de cours 2003, Ecole Normale Supérieure. http://www.math.u-psud.fr/ paulin/notescours

[Bou] Bourdon, M.: Structure conforme au bord et flot géodésique d'un CAT(-1) espace. L'Ens. Math. 41, 63-102 (1995)

[Bow] Bowditch, B.: Geometrical finiteness with variable negative curvature. Duke Math. J. 77, 229274 (1995)

[BR] Bowen, R., Ruelle, D.: The ergodic theory of Axiom A flows. Invent. Math. 29, 181-202 (1975)

[Cou] Coudène, Y.: Gibbs measures on negatively curved manifolds. J. Dynam. Control Syst. 9, 89101 (2003)

[Dal1] Dal'Bo, F.: Remarques sur le spectre des longueurs d'une surface et comptage. Bol. Soc. Bras. Math. 30, 199-221 (1999)

[Dal2] Dal'Bo, F.: Topologie du feuilletage fortement stable. Ann. Inst. Fourier 50, 981-993 (2000)

[FSU] Fishman, L., Simmons, D.S., Urbański. Diophantine approximation and the geometry of limit sets in Gromov hyperbolic metric spaces. arXiv:1301.5630v11, to appear in Memoirs of AMS

[Ham1] Hamenstädt, U.: An explicit description of harmonic measure. Math. Z. 205, 487-499 (1990)

[Ham2] Hamenstädt, U.: Cocycles, hausdorff measures and cross ratios. Erg. Theo. Dyn. Sys. 17, 10611081 (1997) 
[HP1] Hersonsky, S., Paulin, F.: Counting orbit points in coverings of negatively curved manifolds and Hausdorff dimension of cusp excursions. Erg. Theo. Dyn. Sys. 24, 803-824 (2004)

[HP2] Hersonsky, S., Paulin, F.: On the almost sure spiraling of geodesics in negatively curved manifolds. J. Diff. Geom. 85, 271-314 (2010)

[Kat] Katok, S.: Fuchsian groups. University of Chicago Press (1992)

$[\mathrm{KH}]$ Katok A., Hasselblatt B.: Introduction to the modern theory of dynamical systems. Ency. Math. App., vol. 54. Camb. Univ. Press (1995)

[Led1] Ledrappier, F.: Ergodic properties of Brownian motion on covers of compact negatively curved manifolds. Bol. Soc. Bras. Math. 19, 115-140 (1988)

[Led2] Ledrappier, F.: A renewal theorem for the distance in negative curvature. In: 'Stochastic analysis' (Ithaca, 1993), Proc. Symp. Pure Math., vol. 57, pp. 351-360. Amer. Math. Soc. (1995)

[Led3] Ledrappier, F.: Structure au bord des variétés à courbure négative. Sém. Théorie Spec. Géom. Grenoble 13, 97-122 (1994-1995)

[Led4] Ledrappier, F.: Entropie et principe variationnel pour le flot géodésique en courbure négative pincée. In 'Géométrie ergodique'. Mono. L'Ens. Math. F. Dal'Bo ed. L’Ens. Math. 43, 117-144 (2013)

[Moh] Mohsen, O.: Le bas du spectre d'une variété hyperbolique est un point selle. Ann. Sci. Éc. Norm. Sup. 40, 191-207 (2007)

[OP] Otal, J.-P., Peigné, M.: Principe variationnel et groupes kleiniens. Duke Math. J. 125, 15-44 (2004)

[PaP1] Parkkonen, J., Paulin, F.: Prescribing the behaviour of geodesics in negative curvature. Geom. Topol. 14, 277-392 (2010)

[PaP2] Parkkonen, J., Paulin, F.: Spiraling spectra of geodesic lines in negatively curved manifolds. Math. Z. 268, 101-142 (2011)

[PaP3] Parkkonen, J., Paulin, F.: Équidistribution, comptage et approximation par irrationnels quadratiques. J. Mod. Dyn. 6, 1-40 (2012)

[Pau] Paulin, F.: On the critical exponent of discrete group of hyperbolic isometries. Differ. Geom. Appl. 7, 231-236 (1997)

[PPS] Paulin, F., Pollicott, M., Schapira B.: Equilibrium states in negative curvature. Astérisque vol. 373, Soc. Math. France (2015)

[Sch] Schapira, B.: On quasi-invariant transverse measures for the horospherical foliation of a negatively curved manifold. Erg. Theo. Dyn. Syst. 24, 227-257 (2004)

[Spr] Sprindžuk, V.G.: Mahler's problem in metric number theory. Transl. Math. Mono., vol. 25. Amer. Math. Soc. (1969)

Communicated by K. Khanin 